\title{
DISTRIBUTION OF LOW-LEVEL NATURAL RADIOACTIVITY IN A POPULATED MARINE REGION OF THE EASTERN MEDITERRANEAN SEA
}

Nikolaos Evangeliou*, Heleny Florou and Panayotis Kritidis

NCSR 'Demokritos', Institute of Nuclear Technology-Radiation Protection, Environmental Radioactivity

Laboratory, 15310, Agia Paraskevi, Athens, Greece

*Corresponding author: nevag@ipta.demokritos.gr

Received December 9 2011, revised March 24 2012, accepted March 262012

\begin{abstract}
The levels of natural radioactivity have been evaluated in the water column of an eastern Mediterranean region (Saronikos Gulf), with respect to the relevant environmental parameters. A novel methodology was used for the determination of natural radionuclides, which substitutes the time-consuming radiochemical analysis, based on an in situ sample preconcentration using ion-selective manganese fibres placed on pumping systems. With regard to the results obtained, ${ }^{238} \mathrm{U}$-series radionuclides were found at the same level or lower than those observed previously in Mediterranean regions indicating the absence of technologically enhanced naturally occurring radioactive material (TENORM) activities in the area. Similar results were observed for the ${ }^{232} \mathrm{Th}$-series radionuclides and ${ }^{40} \mathrm{~K}$ in the water column in comparison with the relevant literature on the Mediterranean Sea. The calculated ratios of ${ }^{238} \mathrm{U}-{ }^{232} \mathrm{Th}$ and ${ }^{40} \mathrm{~K}-{ }^{232} \mathrm{Th}$ verified the lack of TENORM contribution in the Saronikos Gulf. Finally, a rough estimation was attempted concerning the residence times of fresh water inputs from a treatment plant of domestic wastes (Waste Water Treatment Plant of Psitalia) showing that fresh waters need a maximum of $15.7 \pm 7.6 \mathrm{~d}$ to be mixed with the open sea water.
\end{abstract}

\section{INTRODUCTION}

The study of natural radioactivity is usually performed in order to gain insight into the present levels of harmful pollutants affecting the environment and/or the living organisms (human and nonhuman biota). It is also important to understand the fate of natural radionuclides in the environment, since such investigations can be useful for both the assessment of the public dose rates and the performance of epidemiological studies, as well as to keep reference-data records, to ascertain possible changes in the environmental radioactivity due to nuclear, industrial and other human activities ${ }^{(1)}$.

Natural radioactivity is the main contributor to the annual dose received by the world's population $^{(2)}$, exposure resulting from radionuclides inherent in the earth's crust and from cosmic rays. Most of the natural radionuclides belong to the natural radioactive decay series of ${ }^{238} \mathrm{U},{ }^{235} \mathrm{U}$ and ${ }^{232} \mathrm{Th}$ and their decay products, while there are also single decay radionuclides, particularly ${ }^{40} \mathrm{~K}$. Gamma radiation emitted from these radionuclides is present in all media mainly because they can get dissolved in water and migrate to surface water reservoirs, leading to the possibility of contaminating foodstuffs following soil-plant transfer, as well as getting ingested by human and non-human biota ${ }^{(3)}$. They can also be suspended in air, and in the case of gaseous radionuclides, can emanate into air, facilitating their incorporation into living bodies through inhalation $^{(4)}$. Both types of exposure depend on the activity concentration of the radionuclide, making radiometric techniques a key instrument in dose-rate calculations.

The present study was carried out in central Greece (eastern Mediterranean Sea), specifically, in a marine region adjacent to the capital city of Athens, named the Saronikos Gulf, which is directly connected to the Aegean Sea (Figure 1). The Saronikos Gulf is one of the most conventionally polluted marine environments in Greece, affected by domestic, industrial and anthropogenic activities. Along its northern part, $>30$ industries are located (oil refineries, steel mills, shipyards, etc.) ${ }^{(5)}$, in conjunction with a Waste Water Treatment Plant (WWTP) located in a small island (Psitalia), that outflows in its west central part and, also, the port of Piraeus, one of the largest in Mediterranean Sea. These enclosed marine regions are usually deposition areas of washout and run-off materials due to human activities around the catchment area. Those materials include radionuclide contaminants related to naturally occurring radioactive materials (NORM) and/or technologically enhanced NORM $(\text { TENORM })^{(6)}$. More specifically, in the Saronikos area, apart from the adjoined terrestrial sources that contribute to the marine environment through erosional processes, an additional source of TENORM is present originating from the phosphate fertiliser plant of Drapetsona (west of the port of Piraeus), 


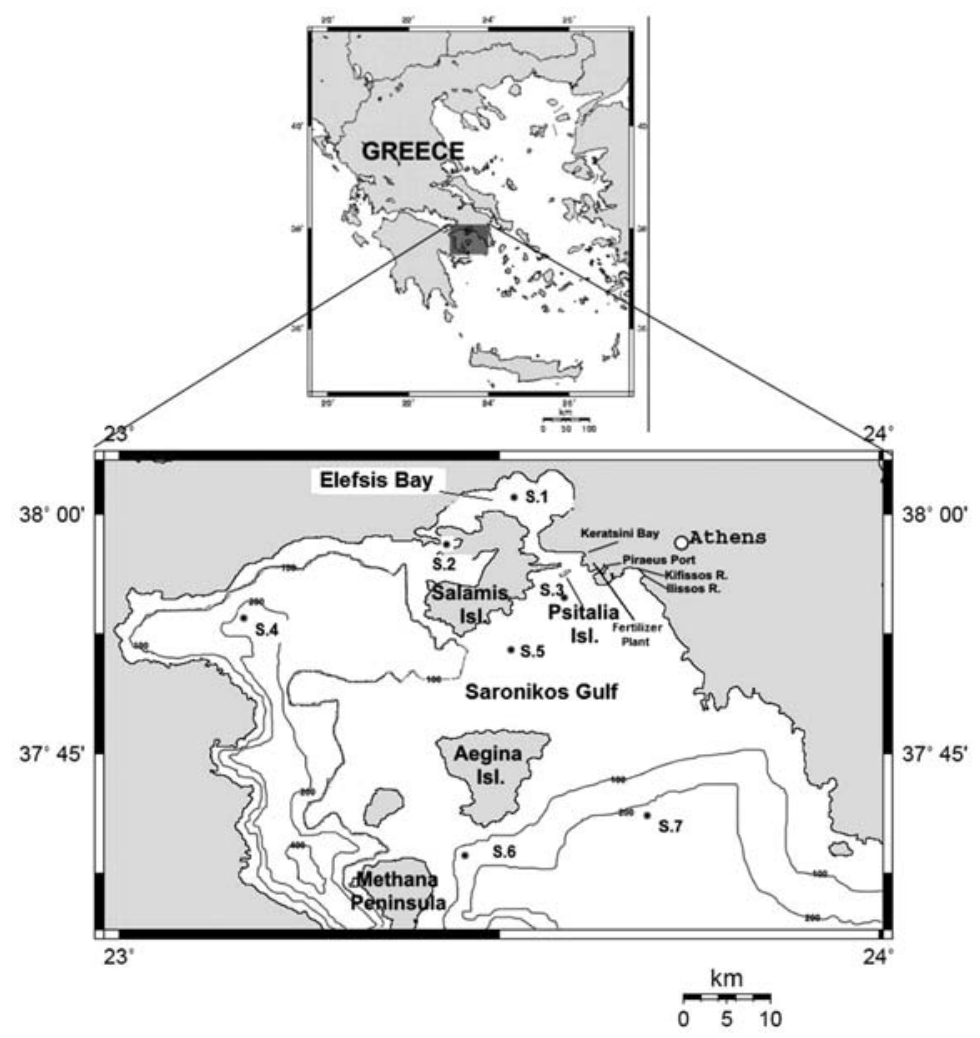

Figure 1. Topography, bathymetry and selected stations (S.1-S.7) of the sampling area (Saronikos Gulf, Greece).

although it has been decommissioned (Figure 1). Stamatis et al. ${ }^{(7)}$ reported increased levels of radioactivity at places, buildings, constructions and materials of the abandoned plant. It is possible that the marine area has been affected by natural radionuclides after transfer through erosional and run-off processes.

To the authors' knowledge, very limited published information is available regarding the levels of natural radioactivity of seawater in the Saronikos Gulf, due to the demanding sampling and analysis of these radionuclides. Therefore, the present work aimed at providing a first radiological study concerning the levels of natural radioactivity and a baseline database for this enclosed ecosystem. The activity concentration levels of the gamma-ray emitting radionuclides in the water column were estimated and subsequently were compared with data from similar marine ecosystems of the Mediterranean Sea. In the present study, the natural occurring radionuclides ${ }^{238} \mathrm{U}$ (calculated from salinity), ${ }^{234} \mathrm{Th}$, ${ }^{226} \mathrm{Ra},{ }^{214} \mathrm{~Pb},{ }^{214} \mathrm{Bi}\left({ }^{238} \mathrm{U}\right.$-series $)$ and ${ }^{232} \mathrm{Th},{ }^{228} \mathrm{Ra}$, ${ }^{228} \mathrm{Th}\left({ }^{232} \mathrm{Th}\right.$-series), as well as ${ }^{40} \mathrm{~K}$ were analysed both spatially and temporally.

\section{MATERIALS AND METHODS}

Challenger Oceanic (one instrument) and McLane (two instruments) large volume in situ pumps ${ }^{(8)}$ were deployed at seven stations (S.1-S.7) in the Saronikos Gulf (two of them-S.1 and S.2-located in a small bay at its northern part, namely the Elefsis Bay) during four seasons (winter 2007, summer 2008, autumn 2008 and winter 2009), in order to filter large volumes of seawater and to collect suspended particles. Sample volumes of 500-1500 1 were processed at flow rates of $0.3-0.5 \mathrm{~m}^{3} \mathrm{~h}^{-1}$. The accuracy of the flow meter was $\pm 2 \%$, operating in a range of $22.7-2730 \mathrm{l} \mathrm{h}^{-1(8)}$. The final depths of the stations were $30 \mathrm{~m}$ (S.1 and S.2), $65 \mathrm{~m}$ (S.3), $97 \mathrm{~m}$ (S.5), $105 \mathrm{~m}$ (S.6) and $220 \mathrm{~m}$ (S.4 and S.7). During the winter 2007 campaign, an inconvenience during sampling resulted in sample collection from only three stations (S.3, S.5 and S.7). Salinity and density were calculated very easily by conductivity measurements using a CTD model YSI 53, which also includes a probe for temperature and $\mathrm{pH}$. The sampling methodology aimed at scavenging radionuclides from seawater using manganese fibres $(\mathrm{Mn}-$ 
fibres). More specifically, cotton wound cartridges, impregnated with manganese oxides in the laboratory, were placed in tandem on the in situ pumping systems to remove dissolved species (such as radium, thorium, plutonium and americium) after a prefilter was used to remove particles from seawater ${ }^{(9-12)}$. This method has the advantage of the sample preconcentration in situ, avoiding the lengthy radiochemical treatment required for such analyses.

The pump separated dissolved and suspended particulate phases via filtration through a pre-cleaned, pre-combusted Whatman GF/A disc filter (pore size of $0.6 \mu \mathrm{m}$, diameter of $142 \mu \mathrm{mm}$ ). It has been found that these filters are easy to handle and they have a high loading capacity and a very low radionuclide blank $^{(13,14)}$.

\section{Impregnation of cartridges with Mn-fibres}

Cotton wound cartridge filters $(25 \mathrm{~cm}$ long and $1 \mu \mathrm{m}$ pore size) were used for impregnation. Impregnation was performed at the laboratory employing a peristaltic pump and by circulating solutions of $\mathrm{KMnO}_{4}$ and $\mathrm{MnCl}_{2} 4 \mathrm{H}_{2} \mathrm{O}$ in a closed loop, in order to react onto the filter. The green-brown precipitate was then formed onto the filter. When the circulating solution was clear, the impregnated filters were removed from the loop, dried in an oven at $60-80^{\circ} \mathrm{C}$ for $24 \mathrm{~h}$ and packed for the sampling ${ }^{(13,14)}$.

\section{Treatment of Mn-fibre cartridges after sampling- gamma spectrometry measurements}

After sampling, the filters were transferred to the laboratory. The disc filters were air-dried, rinsed with distilled water to remove the residual salts and directly measured by gamma spectrometry after transfer into a calibrated measurement pot $(14.2 \mathrm{~cm}$ diameter and $1.0 \mathrm{~cm}$ height). The cartridges were dried and ashed in ovens at $350^{\circ} \mathrm{C}$. The remaining ash was transferred into calibrated measurement pots $(6.8 \mathrm{~cm}$ in diameter and $1.8 \mathrm{~cm}$ in height) for gamma-spectrometry measurements. Prior to the measurements, the samples in the measuring pots were carefully sealed in order to be isolated from the environment and let to reach radioactive equilibrium for $20 \mathrm{~d}$.

The measurements were carried out in a gamma spectrometry system comprising of a high-purity germanium (HPGe) detector (Canberra Coaxial HPGe Detector System) with a relative efficiency of $90 \%$ and resolution of $2.1 \mathrm{keV}$ (at 1332-keV photopeak of ${ }^{60} \mathrm{Co}$ ). The HPGe detector is connected to an 8kmultichannel analyser and the whole system is controlled using Canberra Genie 2000 software. The duration of each measurement was at least $7 \times 10^{4} \mathrm{~s}$ usually overnight.
The energy calibration was performed using standard sources of ${ }^{241} \mathrm{Am}$ and ${ }^{60} \mathrm{Co}$ in a range of $2000 \mathrm{keV}$ in $8 \mathrm{k}$ channels $\left(0.25 \mathrm{keV} \mathrm{ch}{ }^{-1}\right)$. The detector's efficiency was calculated (in connection with energy) using a mixed standard solution of ${ }^{241} \mathrm{Am}$, ${ }^{109} \mathrm{Cd},{ }^{139} \mathrm{Ce},{ }^{57} \mathrm{Co},{ }^{60} \mathrm{Co},{ }^{137} \mathrm{Cs},{ }^{203} \mathrm{Hg},{ }^{113} \mathrm{Sn},{ }^{85} \mathrm{Sr}$, ${ }^{88} \mathrm{Y}$ with a total activity of $5202 \mathrm{~Bq}$. Self-absorption was assumed to be negligible as the geometries used in the study were of small volume $(6.8 \mathrm{~cm}$ in diameter and 1.8 in $\mathrm{cm}$ height for the ashed cartridges used for dissolved fractions and $14.2 \mathrm{~cm}$ in diameter and $1.0 \mathrm{~cm}$ in height for the disc filters used for the particulates) and efficiency calibration was performed using the same geometry. For justification, the self-absorption of the samples was checked using the Monte Carlo efficiency transfer (code DETEFF) and it was found insignificant. The quality assurance of the measurements is done here periodically, checked by participating in proficiency tests (e.g. within 2010 ERL has participated in two tests, the IAEA-CRP1471-01 proficiency test and an EC inter-laboratory comparison JRC-IRMM).

The activity concentrations of the dissolved fractions of gamma-emitting natural radionuclides were estimated taking into account the activities of the first and the second $\mathrm{MnO}_{2}$ cartridge, respectively, as well as the collection efficiency $(E)$ of the filters using the following equation:

$$
A(\mathrm{rad})=\frac{A_{1}}{E}=\frac{A_{1}}{1-A_{2} / A_{1}}
$$

where $A_{1}$ and $A_{2}$ are the activities of each analysed radionuclide in the first and the second cartridge ${ }^{(14)}$. The average collection efficiencies $(E)$ of the cartridges used are shown in Table 1. Efficiencies were kept over $50 \%$ for all the radionuclides analysed. Previous studies have shown that collection efficiencies $<50 \%$ usually result in extremely large activities posing additional errors. The uncertainties of the activity concentrations in the first and the second filter $\left(A_{1}, A_{2}\right)$ were $1 \sigma$ values derived from counting statistics (typical counting statistic error: $8 \%$ ). The final results presented in this study were obtained by concatenating the activities of the disc filters (used for particulates) with those of cartridges (corresponding to the dissolved fraction), whereas the final uncertainty given was estimated by propagation. Table 2 depicts the specific radionuclides, as well as their peak energies and intensities used in the calculations done here.

\footnotetext{
${ }^{238} \mathrm{U}$ and ${ }^{40} \mathrm{~K}$ activity calculations

${ }^{238} \mathrm{U}$ was calculated via its proportionality with salinity ( $S$, psu). Typically, the relationship derived by Chen et al. ${ }^{(15)}$ is used in open ocean studies.
} 
N. EVANGELIOU ET AL.

Table 1. Percentage estimated average efficiency of the $\mathrm{MnO}_{2}$ filters used for radionuclide scavenging during the four seasons of sampling.

\begin{tabular}{lccccccc}
\hline Period & ${ }^{234} \mathrm{Th}(\%)$ & ${ }^{226} \mathrm{Ra}(\%)$ & ${ }^{214} \mathrm{~Pb}(\%)$ & ${ }^{214} \mathrm{Bi}(\%)$ & ${ }^{232} \mathrm{Th}(\%)$ & ${ }^{228} \mathrm{Ra}(\%)$ & ${ }^{228} \mathrm{Th}(\%)$ \\
\hline Winter 2007 & $58 \pm 25$ & $64 \pm 16$ & $77 \pm 18$ & $79 \pm 18$ & $61 \pm 14$ & $77 \pm 14$ & $74 \pm 16$ \\
Summer 2008 & $58 \pm 12$ & $70 \pm 16$ & $72 \pm 18$ & $77 \pm 21$ & $62 \pm 15$ & $72 \pm 17$ & $69 \pm 21$ \\
Autumn 2008 & $68 \pm 11$ & $79 \pm 16$ & $70 \pm 17$ & $86 \pm 20$ & $72 \pm 14$ & $71 \pm 16$ & $67 \pm 20$ \\
Winter 2009 & $59 \pm 10$ & $78 \pm 14$ & $78 \pm 15$ & $65 \pm 19$ & $81 \pm 14$ & $70 \pm 15$ & $65 \pm 18$ \\
\hline
\end{tabular}

Standard deviations of the respective runs are also included.

However, ${ }^{238} \mathrm{U}$ concentrations in the Mediterranean are enhanced relative to the U-salinity relationship of Chen et al. ${ }^{(15)}$, due to phosphate fertiliser run-off into this enclosed basin. Although $\mathrm{U}$ can deviate from the conservative behaviour in some coastal areas $^{(16-18)}$, this deviation occurs usually where either fresh water or reducing conditions dominate. The salinity range observed in the present study $(32.8-39.2 \mathrm{psu})$ indicates that fresh water supplies have no significant influence in salinity $(S)$, although there are fresh water inputs in the system (ephemeral streams and WWTP). Therefore, ${ }^{238} \mathrm{U}$ concentrations $\left(\mathrm{Bq} \mathrm{m} \mathrm{m}^{-3}\right)$ were derived from the following equation, which has been found close to real-time measurements in the Mediterranean waters ${ }^{(19)}$.

$$
{ }^{238} \mathrm{U}=(1.1775 \pm 0.0243) \times S
$$

Stable potassium constitutes $1.1 \%$ of the salts that are diluted in seawater. Tsabaris and Ballas ${ }^{(20)}$ reported that the mass concentration (in $\mathrm{g} \mathrm{m}^{-3}$ ) of the stable potassium, $C(K)$, relative to the salinity $S$ (in psu) and density $D$ (in $\mathrm{kg} \mathrm{m}^{-3}$ ) of the seawater, is given by the following equation (see also: Supplementary data ):

$$
C(K)=\frac{D \times S}{90.9}
$$

On the other hand, the abundance of ${ }^{40} \mathrm{~K}$ is $0.0117 \%$, so that the volumetric concentration (in $\mathrm{g} \mathrm{m}^{-3}$ ) of ${ }^{40} \mathrm{~K}$ is given in the following equation:

$$
C\left({ }^{40} K\right)=\frac{0.0117}{100} \times C(K)
$$

The activity concentrations of ${ }^{40} \mathrm{~K}\left(\mathrm{~Bq} \mathrm{~m}^{-3}\right)$ are given by the following equation (see also: Supplementary data):

$$
A\left(\mathrm{~Bq} \mathrm{~m}^{-3}\right)=\frac{\ln 2}{t_{1 / 2}} \frac{C\left({ }^{40} K\right)}{M} N_{\mathrm{A}}
$$

where, $t_{1 / 2}$ is the half-life of ${ }^{40} \mathrm{~K}\left(=1.28 \times 10^{9} \mathrm{y}\right.$ or $\left.1.28 \times 10^{9} \times 365 \times 24 \times 3600 \mathrm{~s}\right), M$ is the atomic weight
Table 2. The energies and the intensities of the most intense gamma rays used to determine radionuclide activity concentrations.

\begin{tabular}{llc}
\hline Radionuclide & \multicolumn{1}{c}{ Energy (keV) } & Intensity (\%) \\
\hline${ }^{234} \mathrm{Th}$ & 63.3 & 5.7 \\
& 92.6 & 6.7 \\
${ }^{226} \mathrm{Ra}$ & 295.2 & 18.9 \\
& 352.5 & 35.0 \\
${ }^{214} \mathrm{~Pb}$ & 609.4 & 44.6 \\
${ }^{214} \mathrm{Bi}$ & 295.2 & 18.9 \\
${ }^{228} \mathrm{Ra}\left({ }^{228} \mathrm{Ac}\right)$ & 352.5 & 35.0 \\
${ }^{228} \mathrm{Th}\left({ }^{212} \mathrm{~Pb}\right.$ and $\left.{ }^{208} \mathrm{Tl}\right)$ & 338.4 & 11.4 \\
${ }^{232} \mathrm{Th}$ & 238.6 & 26.6 \\
& 583.1 & 43.5 \\
& From ${ }^{228} \mathrm{Ra}$ to ${ }^{228} \mathrm{Th}$ & 30.6 \\
\hline
\end{tabular}

of ${ }^{40} \mathrm{~K}(=40 \mathrm{~g}), N_{\mathrm{A}}$ is the Avogadro number (= $6.023 \times 10^{23}$ molecules $\mathrm{mol}^{-1}$ ) and $S$ is the salinity data obtained by the in situ CTD probe (in psu).

Equation (5) has been tested by real-time measurements in the open sea and it gives the qualitative and quantitative results of ${ }^{40} \mathrm{~K}$ in seawater ${ }^{(20)}$.

\section{RESULTS AND DISCUSSION}

${ }^{238} \mathrm{U}$-series radionuclides $\left({ }^{234} \mathrm{Th},{ }^{226} \mathrm{Ra},{ }^{214} \mathrm{~Pb}\right.$ and ${ }^{214} \mathrm{Bi}$ )

${ }^{238} \mathrm{U}$-series radionuclides $\left({ }^{234} \mathrm{Th},{ }^{226} \mathrm{Ra},{ }^{214} \mathrm{~Pb}\right.$ and ${ }^{214} \mathrm{Bi}$ ) were quantitatively determined in seawater. ${ }^{238} \mathrm{U}$ activity concentrations were estimated by salinity values using the equation reported by Delanghe et al. ${ }^{(19)}$. The respective results are shown in Table 3 and illustrated in Figure 2.

${ }^{238} \mathrm{U}$ activity concentrations ranged between $39.0 \pm 0.8$ and $45.2 \pm 0.9 \mathrm{~Bq} \mathrm{~m}^{-3}$ depending on the depth during winter 2007, between $38.0 \pm 0.9$ and $45.8 \pm 0.9 \mathrm{~Bq} \mathrm{~m}^{-3}$ during summer 2008 , between $42.6 \pm 0.9$ and $46.3 \pm 0.9 \mathrm{~Bq} \mathrm{~m}^{-3}$ during autumn 2008 and between $44.7 \pm 0.9$ and $46.2 \pm 1.0 \mathrm{~Bq} \mathrm{~m}^{-3}$ during winter 2009 (Table 3). It is noteworthy that ${ }^{238} \mathrm{U}$ activity concentrations remained relatively 
DISTRIBUTION OF LOW-LEVEL NATURAL RADIOACTIVITY

Table 3. ${ }^{238} \mathrm{U}$ series radionuclides (salinity-derived ${ }^{238} \mathrm{U},{ }^{234} \mathrm{Th},{ }^{226} \mathrm{Ra},{ }^{214} \mathrm{~Pb}$ and ${ }^{214} \mathrm{Bi}$ ) in the seawater column of the stations in the Saronikos Gulf during the different sampling campaigns.

\begin{tabular}{|c|c|c|c|c|c|c|}
\hline & Salinity (psu) & ${ }^{238} \mathrm{U}\left(\mathrm{Bq} \mathrm{m}^{-3}\right)$ & ${ }^{234} \mathrm{Th}\left(\mathrm{Bq} \mathrm{m}{ }^{-3}\right)$ & ${ }^{226} \mathrm{Ra}\left(\mathrm{Bq} \mathrm{m}{ }^{-3}\right)$ & ${ }^{214} \mathrm{~Pb}\left(\mathrm{~Bq} \mathrm{~m} \mathrm{~m}^{-3}\right)$ & ${ }^{214} \mathrm{Bi}\left(\mathrm{Bq} \mathrm{m}^{-3}\right)$ \\
\hline \multicolumn{7}{|l|}{ Winter 2007} \\
\hline $\mathrm{S} .3(0 \mathrm{~m})$ & 36.7 & $43.2 \pm 0.9$ & $27.5 \pm 0.6$ & $4.4 \pm 0.5$ & $4.4 \pm 0.2$ & $2.9 \pm 0.5$ \\
\hline $\mathrm{S} .3(15 \mathrm{~m})$ & 36.4 & $42.9 \pm 0.9$ & $30.0 \pm 0.2$ & $3.8 \pm 0.1$ & $2.2 \pm 0.1$ & $3.4 \pm 0.1$ \\
\hline S.3 $(60 \mathrm{~m})$ & 33.1 & $39.0 \pm 0.8$ & $15.7 \pm 1.6$ & $1.3 \pm 0.1$ & $1.7 \pm 0.4$ & $0.9 \pm 0.1$ \\
\hline $\mathrm{S} .5(0 \mathrm{~m})$ & 38.1 & $44.9 \pm 0.9$ & $13.2 \pm 2.1$ & $1.9 \pm 0.3$ & $2.3 \pm 0.4$ & $1.9 \pm 0.4$ \\
\hline S. $5(35 \mathrm{~m})$ & 37.9 & $44.6 \pm 0.9$ & $12.1 \pm 2.1$ & $0.3 \pm 0.1$ & $1.0 \pm 0.1$ & $1.1 \pm 0.1$ \\
\hline S. $5(95 \mathrm{~m})$ & 38.4 & $45.2 \pm 0.9$ & $17.0 \pm 1.1$ & $2.8 \pm 0.3$ & $1.5 \pm 0.1$ & $2.1 \pm 0.1$ \\
\hline $\mathrm{S} .7(0 \mathrm{~m})$ & 38.4 & $45.2 \pm 0.9$ & $28.2 \pm 2.4$ & $4.1 \pm 0.1$ & $2.4 \pm 0.1$ & $4.2 \pm 0.3$ \\
\hline S.7 $(50 \mathrm{~m})$ & 38.1 & $44.9 \pm 0.9$ & $56.0 \pm 5.3$ & $2.5 \pm 0.3$ & $1.8 \pm 0.5$ & $3.2 \pm 0.2$ \\
\hline S.7 (100 m) & 37.7 & $44.4 \pm 0.9$ & $37.3 \pm 0.9$ & $4.8 \pm 0.1$ & $2.5 \pm 0.1$ & $2.6 \pm 0.1$ \\
\hline S.7 (200 m) & 36.5 & $43.0 \pm 0.9$ & $17.4 \pm 1.4$ & $1.2 \pm 0.3$ & $1.2 \pm 0.3$ & $2.2 \pm 0.2$ \\
\hline \multicolumn{7}{|l|}{ Summer 2008} \\
\hline $\mathrm{S} .1(0 \mathrm{~m})$ & 38.5 & $45.3 \pm 0.9$ & $14.9 \pm 2.1$ & $2.6 \pm 1.3$ & $3.8 \pm 0.3$ & $3.0 \pm 0.8$ \\
\hline S. $1(15 \mathrm{~m})$ & 34.2 & $40.3 \pm 0.8$ & $43.1 \pm 1.1$ & $9.0 \pm 0.8$ & $4.2 \pm 0.2$ & $8.0 \pm 0.5$ \\
\hline S. $1(20 \mathrm{~m})$ & 34.0 & $40.0 \pm 0.8$ & $12.7 \pm 2.6$ & $1.0 \pm 0.2$ & $1.4 \pm 0.3$ & $0.7 \pm 0.1$ \\
\hline $\mathrm{S} .2(0 \mathrm{~m})$ & 38.9 & $45.8 \pm 0.9$ & $17.6 \pm 2.7$ & $0.2 \pm 0.1$ & $0.4 \pm 0.1$ & $0.2 \pm 0.1$ \\
\hline S.2 $(15 \mathrm{~m})$ & 34.1 & $40.2 \pm 0.8$ & $17.1 \pm 1.5$ & $2.7 \pm 0.3$ & $2.7 \pm 0.5$ & $0.7 \pm 0.1$ \\
\hline $\mathrm{S} .2(20 \mathrm{~m})$ & 33.9 & $39.9 \pm 0.8$ & $6.0 \pm 2.2$ & $1.1 \pm 0.1$ & $1.3 \pm 0.1$ & $1.1 \pm 0.4$ \\
\hline $\mathrm{S} .3(0 \mathrm{~m})$ & 38.7 & $45.6 \pm 0.9$ & $14.4 \pm 4.6$ & $0.6 \pm 0.2$ & $0.6 \pm 0.2$ & $1.2 \pm 0.1$ \\
\hline S.3 $(15 \mathrm{~m})$ & 34.1 & $40.2 \pm 0.8$ & $15.1 \pm 3.9$ & $1.9 \pm 0.5$ & $1.6 \pm 0.1$ & $0.8 \pm 0.1$ \\
\hline $\mathrm{S} .3(60 \mathrm{~m})$ & 32.3 & $38.0 \pm 0.8$ & $28.4 \pm 2.8$ & $6.1 \pm 1.1$ & $20.1 \pm 5.1$ & $20.2 \pm 5.4$ \\
\hline $\mathrm{S} .4(0 \mathrm{~m})$ & 38.2 & $45.0 \pm 0.9$ & $50.7 \pm 6.8$ & $0.3 \pm 0.1$ & $0.3 \pm 0.1$ & $0.2 \pm 0.1$ \\
\hline $\mathrm{S} .4(50 \mathrm{~m})$ & 33.9 & $39.9 \pm 0.8$ & $22.2 \pm 2.6$ & $0.3 \pm 0.1$ & $0.3 \pm 0.1$ & $0.3 \pm 0.1$ \\
\hline $\mathrm{S} .4(100 \mathrm{~m})$ & 33.7 & $39.7 \pm 0.8$ & $25.7 \pm 3.6$ & $0.5 \pm 0.1$ & $0.5 \pm 0.2$ & $0.5 \pm 0.1$ \\
\hline $\mathrm{S} .4(200 \mathrm{~m})$ & 33.3 & $39.2 \pm 0.8$ & $51.0 \pm 0.4$ & $0.7 \pm 0.2$ & $0.7 \pm 0.3$ & $0.7 \pm 0.2$ \\
\hline $\mathrm{S} .5(0 \mathrm{~m})$ & 38.3 & $45.1 \pm 0.9$ & $21.4 \pm 0.1$ & $3.4 \pm 0.3$ & $3.0 \pm 0.5$ & $5.4 \pm 0.1$ \\
\hline S. $5(35 \mathrm{~m})$ & 34.5 & $40.6 \pm 0.8$ & $13.5 \pm 0.9$ & $0.2 \pm 0.1$ & $0.1 \pm 0.1$ & $0.2 \pm 0.1$ \\
\hline S.5 (95 m) & 32.8 & $38.6 \pm 0.8$ & $42.7 \pm 1.4$ & $2.1 \pm 0.2$ & $2.5 \pm 0.6$ & $3.2 \pm 0.3$ \\
\hline S. $6(0 \mathrm{~m})$ & 38.1 & $44.9 \pm 0.9$ & $54.5 \pm 10.1$ & $0.5 \pm 0.1$ & $0.5 \pm 0.1$ & $0.5 \pm 0.1$ \\
\hline S. $6(35 \mathrm{~m})$ & 35.0 & $41.2 \pm 0.9$ & $26.1 \pm 4.2$ & $0.4 \pm 0.1$ & $0.4 \pm 0.1$ & $0.3 \pm 0.1$ \\
\hline S.6 $(100 \mathrm{~m})$ & 34.2 & $40.3 \pm 0.8$ & $58.7 \pm 3.8$ & $0.4 \pm 0.1$ & $0.3 \pm 0.1$ & $0.4 \pm 0.1$ \\
\hline $\mathrm{S} .7(0 \mathrm{~m})$ & 37.8 & $44.5 \pm 0.9$ & $48.0 \pm 2.8$ & $0.4 \pm 0.1$ & $0.3 \pm 0.1$ & $0.4 \pm 0.1$ \\
\hline $\mathrm{S} .7(50 \mathrm{~m})$ & 34.1 & $40.2 \pm 0.8$ & $27.3 \pm 2.7$ & $0.2 \pm 0.1$ & $0.2 \pm 0.1$ & $0.2 \pm 0.1$ \\
\hline S.7 (100 m) & 33.9 & $39.9 \pm 0.8$ & $51.6 \pm 0.9$ & $0.2 \pm 0.1$ & $0.2 \pm 0.1$ & $0.2 \pm 0.1$ \\
\hline S.7 $(200 \mathrm{~m})$ & 33.7 & $39.7 \pm 0.8$ & $23.8 \pm 3.3$ & $0.1 \pm 0.1$ & $0.1 \pm 0.1$ & $0.1 \pm 0.1$ \\
\hline \multicolumn{7}{|l|}{ Autumn 2008} \\
\hline $\mathrm{S} .1(0 \mathrm{~m})$ & 38.9 & $45.8 \pm 0.9$ & $8.9 \pm 0.8$ & $0.6 \pm 0.1$ & $0.4 \pm 0.1$ & $0.4 \pm 0.1$ \\
\hline $\mathrm{S} .1(15 \mathrm{~m})$ & 38.6 & $45.5 \pm 0.9$ & $8.3 \pm 2.2$ & $0.8 \pm 0.1$ & $0.7 \pm 0.2$ & $0.7 \pm 0.1$ \\
\hline $\mathrm{S} .1(20 \mathrm{~m})$ & 38.5 & $45.3 \pm 0.9$ & $28.2 \pm 5.3$ & $1.2 \pm 0.3$ & $1.3 \pm 0.4$ & $1.2 \pm 0.1$ \\
\hline $\mathrm{S} .2(0 \mathrm{~m})$ & 37.2 & $43.8 \pm 0.9$ & $24.5 \pm 4.5$ & $0.9 \pm 0.1$ & $0.9 \pm 0.1$ & $0.7 \pm 0.2$ \\
\hline S.2 $(15 \mathrm{~m})$ & 36.2 & $42.6 \pm 0.9$ & $22.4 \pm 2.4$ & $1.6 \pm 0.3$ & $1.1 \pm 0.1$ & $0.9 \pm 0.1$ \\
\hline S.2 $(20 \mathrm{~m})$ & 37.0 & $43.6 \pm 0.9$ & $27.6 \pm 0.3$ & $2.6 \pm 0.3$ & $2.6 \pm 0.5$ & $3.8 \pm 1.0$ \\
\hline $\mathrm{S} .3(0 \mathrm{~m})$ & 38.7 & $45.6 \pm 0.9$ & $35.1 \pm 2.5$ & $1.8 \pm 0.2$ & $2.6 \pm 0.1$ & $0.9 \pm 0.1$ \\
\hline $\mathrm{S} .3(15 \mathrm{~m})$ & 37.8 & $44.5 \pm 0.9$ & $43.7 \pm 1.0$ & $1.7 \pm 0.4$ & $2.1 \pm 0.3$ & $1.8 \pm 0.3$ \\
\hline $\mathrm{S} .3(60 \mathrm{~m})$ & 36.2 & $42.6 \pm 0.9$ & $32.6 \pm 2.7$ & $3.7 \pm 0.1$ & $2.0 \pm 0.1$ & $2.7 \pm 0.4$ \\
\hline $\mathrm{S} .4(0 \mathrm{~m})$ & 38.4 & $45.2 \pm 0.9$ & $32.2 \pm 0.5$ & $0.8 \pm 0.2$ & $0.7 \pm 0.2$ & $1.0 \pm 0.2$ \\
\hline $\mathrm{S} .4(50 \mathrm{~m})$ & 38.2 & $45.0 \pm 0.9$ & $56.8 \pm 2.4$ & $1.0 \pm 0.2$ & $1.1 \pm 0.2$ & $0.9 \pm 0.2$ \\
\hline $\mathrm{S} .4(100 \mathrm{~m})$ & 38.3 & $45.1 \pm 0.9$ & $46.7 \pm 2.9$ & $0.6 \pm 0.1$ & $0.6 \pm 0.2$ & $0.9 \pm 0.2$ \\
\hline $\mathrm{S} .4(200 \mathrm{~m})$ & 38.9 & $45.8 \pm 0.9$ & $50.1 \pm 2.7$ & $3.6 \pm 0.3$ & $2.7 \pm 0.1$ & $3.2 \pm 0.6$ \\
\hline $\mathrm{S} .5(0 \mathrm{~m})$ & 38.0 & $44.7 \pm 0.9$ & $56.8 \pm 4.8$ & $8.2 \pm 0.8$ & $6.7 \pm 0.5$ & $4.6 \pm 0.1$ \\
\hline S. $5(35 \mathrm{~m})$ & 38.8 & $45.7 \pm 0.9$ & $27.1 \pm 3.7$ & $1.6 \pm 0.3$ & $1.7 \pm 0.2$ & $1.5 \pm 0.3$ \\
\hline S. $5(95 \mathrm{~m})$ & 38.5 & $45.3 \pm 0.9$ & $18.8 \pm 1.0$ & $1.8 \pm 0.1$ & $1.9 \pm 0.1$ & $1.6 \pm 0.4$ \\
\hline S. $6(0 \mathrm{~m})$ & 38.5 & $45.3 \pm 0.9$ & $40.0 \pm 2.0$ & $0.6 \pm 0.2$ & $0.7 \pm 0.1$ & $0.7 \pm 0.2$ \\
\hline S.6 (35 m) & 39.0 & $45.9 \pm 0.9$ & $25.1 \pm 3.8$ & $1.4 \pm 0.1$ & $1.4 \pm 0.3$ & $1.3 \pm 0.2$ \\
\hline S.6 (100 m) & 37.9 & $44.6 \pm 0.9$ & $26.8 \pm 2.7$ & $1.6 \pm 0.4$ & $1.7 \pm 0.3$ & $1.6 \pm 0.4$ \\
\hline $\mathrm{S} .7(0 \mathrm{~m})$ & 38.9 & $45.8 \pm 0.9$ & $29.6 \pm 2.9$ & $1.0 \pm 0.2$ & $1.0 \pm 0.2$ & $1.0 \pm 0.2$ \\
\hline $\mathrm{S} .7(50 \mathrm{~m})$ & 38.6 & $45.5 \pm 0.9$ & $28.8 \pm 4.7$ & $1.4 \pm 0.1$ & $1.7 \pm 0.1$ & $0.3 \pm 0.1$ \\
\hline
\end{tabular}

Continued 
Table 3. Continued

\begin{tabular}{|c|c|c|c|c|c|c|}
\hline & Salinity (psu) & ${ }^{238} \mathrm{U}\left(\mathrm{Bq} \mathrm{m}^{-3}\right)$ & ${ }^{234} \mathrm{Th}\left(\mathrm{Bq} \mathrm{m}{ }^{-3}\right)$ & ${ }^{226} \mathrm{Ra}\left(\mathrm{Bq} \mathrm{m}{ }^{-3}\right)$ & ${ }^{214} \mathrm{~Pb}\left(\mathrm{~Bq} \mathrm{~m}{ }^{-3}\right)$ & ${ }^{214} \mathrm{Bi}\left(\mathrm{Bq} \mathrm{m}^{-3}\right)$ \\
\hline $\mathrm{S} .7(100 \mathrm{~m})$ & 38.6 & $45.5 \pm 0.9$ & $30.4 \pm 6.3$ & $1.2 \pm 0.3$ & $1.2 \pm 0.2$ & $1.4 \pm 0.1$ \\
\hline $\mathrm{S} .7(200 \mathrm{~m})$ & 39.3 & $46.3 \pm 1.0$ & $30.3 \pm 4.4$ & $0.4 \pm 0.2$ & $0.4 \pm 0.2$ & $0.3 \pm 0.1$ \\
\hline \multicolumn{7}{|l|}{ Winter 2009} \\
\hline $\mathrm{S} .1(0 \mathrm{~m})$ & 38.6 & $45.5 \pm 0.9$ & $17.0 \pm 4.0$ & $2.3 \pm 0.3$ & $1.9 \pm 0.4$ & $2.8 \pm 0.1$ \\
\hline S. $1(15 \mathrm{~m})$ & 38.1 & $44.9 \pm 0.9$ & $15.7 \pm 2.8$ & $1.9 \pm 0.1$ & $2.0 \pm 0.3$ & $2.6 \pm 0.4$ \\
\hline S. $1(20 \mathrm{~m})$ & 38.9 & $45.8 \pm 0.9$ & $9.0 \pm 4.8$ & $1.4 \pm 0.3$ & $1.5 \pm 0.2$ & $1.3 \pm 0.2$ \\
\hline $\mathrm{S} .2(0 \mathrm{~m})$ & 38.3 & $45.1 \pm 0.9$ & $7.2 \pm 2.5$ & $0.4 \pm 0.1$ & $0.4 \pm 0.1$ & $0.4 \pm 0.1$ \\
\hline $\mathrm{S} .2(15 \mathrm{~m})$ & 38.0 & $44.7 \pm 0.9$ & $12.1 \pm 4.2$ & $0.8 \pm 0.2$ & $0.7 \pm 0.2$ & $0.8 \pm 0.2$ \\
\hline $\mathrm{S} .2(20 \mathrm{~m})$ & 38.5 & $45.3 \pm 0.9$ & $13.7 \pm 2.0$ & $1.3 \pm 0.2$ & $1.3 \pm 0.1$ & $1.3 \pm 0.1$ \\
\hline $\mathrm{S} .3(0 \mathrm{~m})$ & 39.2 & $46.2 \pm 1.0$ & $33.3 \pm 3.3$ & $1.4 \pm 0.3$ & $1.2 \pm 0.3$ & $5.0 \pm 0.3$ \\
\hline $\mathrm{S} .3(15 \mathrm{~m})$ & 38.9 & $45.8 \pm 0.9$ & $33.6 \pm 3.8$ & $1.0 \pm 0.2$ & $0.7 \pm 0.2$ & $0.9 \pm 0.2$ \\
\hline $\mathrm{S} .3(60 \mathrm{~m})$ & 38.5 & $45.3 \pm 0.9$ & $36.2 \pm 1.0$ & $0.5 \pm 0.1$ & $0.6 \pm 0.2$ & $0.5 \pm 0.1$ \\
\hline $\mathrm{S} .4(0 \mathrm{~m})$ & 38.6 & $45.5 \pm 0.9$ & $37.0 \pm 10.8$ & $1.4 \pm 0.5$ & $1.8 \pm 0.1$ & $0.9 \pm 0.2$ \\
\hline $\mathrm{S} .4(50 \mathrm{~m})$ & 38.8 & $45.7 \pm 0.9$ & $32.5 \pm 5.4$ & $1.3 \pm 0.4$ & $1.4 \pm 0.3$ & $1.1 \pm 0.1$ \\
\hline $\mathrm{S} .4(100 \mathrm{~m})$ & 39.1 & $46.0 \pm 1.0$ & $22.3 \pm 1.3$ & $1.5 \pm 0.3$ & $1.5 \pm 0.1$ & $1.5 \pm 0.1$ \\
\hline $\mathrm{S} .4(200 \mathrm{~m})$ & 39.2 & $46.2 \pm 1.0$ & $32.9 \pm 6.7$ & $2.2 \pm 0.6$ & $2.3 \pm 0.2$ & $2.0 \pm 0.5$ \\
\hline $\mathrm{S} .5(0 \mathrm{~m})$ & 39.1 & $46.0 \pm 1.0$ & $49.3 \pm 3.6$ & $0.8 \pm 0.1$ & $0.8 \pm 0.1$ & $0.6 \pm 0.2$ \\
\hline $\mathrm{S} .5(35 \mathrm{~m})$ & 39.0 & $45.9 \pm 0.9$ & $24.3 \pm 6.5$ & $1.3 \pm 0.4$ & $1.4 \pm 0.3$ & $1.3 \pm 0.1$ \\
\hline $\mathrm{S} .5(95 \mathrm{~m})$ & 39.0 & $45.9 \pm 0.9$ & $37.0 \pm 1.5$ & $0.7 \pm 0.2$ & $0.8 \pm 0.2$ & $0.6 \pm 0.2$ \\
\hline $\mathrm{S} .6(0 \mathrm{~m})$ & 38.5 & $45.3 \pm 0.9$ & $22.6 \pm 2.5$ & $0.9 \pm 0.1$ & $0.9 \pm 0.5$ & $1.1 \pm 0.4$ \\
\hline S.6 $(35 \mathrm{~m})$ & 38.8 & $45.7 \pm 0.9$ & $23.3 \pm 2.4$ & $1.2 \pm 0.1$ & $1.0 \pm 0.2$ & $4.1 \pm 0.2$ \\
\hline S.6 (100 m) & 39.1 & $46.0 \pm 1.0$ & $40.5 \pm 6.2$ & $1.4 \pm 0.1$ & $1.9 \pm 0.3$ & $1.4 \pm 0.1$ \\
\hline $\mathrm{S} .7(0 \mathrm{~m})$ & 39.0 & $45.9 \pm 0.9$ & $32.3 \pm 1.2$ & $1.3 \pm 0.2$ & $1.4 \pm 0.3$ & $1.4 \pm 0.4$ \\
\hline S.7 $(50 \mathrm{~m})$ & 39.1 & $46.0 \pm 1.0$ & $32.8 \pm 2.3$ & $2.1 \pm 0.1$ & $2.0 \pm 0.2$ & $2.9 \pm 0.2$ \\
\hline S.7 (100 m) & 38.7 & $45.6 \pm 0.9$ & $50.2 \pm 12.0$ & $2.0 \pm 0.2$ & $1.9 \pm 0.4$ & $2.2 \pm 0.5$ \\
\hline S.7 (200 m) & 39.0 & $45.9 \pm 0.9$ & $36.5 \pm 3.3$ & $1.4 \pm 0.2$ & $1.4 \pm 0.4$ & $1.3 \pm 0.1$ \\
\hline
\end{tabular}

The final uncertainties were calculated by propagation.

stable throughout the water column and during the different sampling seasons, as a result of the slight variation of the salinity since the area is characterised by small fresh water inputs (only few streams outflow into the Gulf). The lowest values of ${ }^{238} \mathrm{U}$ were found at a depth of $60 \mathrm{~m} \mathrm{~S} .3$, where the major fresh water inputs are presented, as low dense waters outflow next to the station, at a depth of $65 \mathrm{~m}$ from the pipelines of the WWTP of Psitalia (Figure 1). The salinity-estimated values of ${ }^{238} \mathrm{U}$ found comparable to others occurred after radiochemical treatment in the eastern Mediterranean region, e.g. $40-50 \mathrm{~Bq} \mathrm{~m}^{-3}$ in the north Aegean ${ }^{(21)}, 44-45$ $\mathrm{Bq} \mathrm{m}^{-3}$ in the south Aegean, $43-45 \mathrm{~Bq} \mathrm{~m}^{-3}$ in the Ligurian Sea and 43-46 in the Tyrrhenian Sea ${ }^{(22)}$.

The activity concentrations of ${ }^{234} \mathrm{Th}$ fluctuated from $12.1 \pm 2.1$ to $56.0 \pm 5.3 \mathrm{~Bq} \mathrm{~m}^{-3}$ in winter 2007, from $12.7 \pm 2.6$ to $58.7 \pm 3.8 \mathrm{~Bq} \mathrm{~m}{ }^{-3}$ in summer 2008 , from $8.3 \pm 2.2$ to $56.8 \pm 4.8 \mathrm{~Bq} \mathrm{~m}^{-3}$ in autumn 2008 and from $7.2 \pm 2.5$ to $50.2 \pm 12.0 \mathrm{~Bq}$ $\mathrm{m}^{-3}$ in winter 2009 depending on the sampling depth (Table 3 and Figure 2). The observed large range of ${ }^{234} \mathrm{Th}$ is attributed to the different nature of this element. ${ }^{234} \mathrm{Th}$ shows high affinity for particles in the marine environment (particle-reactive element). It is produced by the radioactive decay of the completely conservative ${ }^{238} \mathrm{U}$ and its radioactive disequilibrium in the marine environment has been used to trace processes occurring over short timescales $^{(23,24)}$. The values observed here are of the same level as those observed in the Mediterranean Sea, although the variation of values is extremely wide. However, some significant activity concentrations of the relevant literature concerning the eastern Mediterranean are: $25-28 \mathrm{~Bq} \mathrm{~m}^{-3}$ in the Ligurian Sea, $36-50 \mathrm{~Bq} \mathrm{~m}^{-3}$ in the Tyrrhenian Sea and $37-43 \mathrm{~Bq} \mathrm{~m}^{-3}$ in the Aegean Sea ${ }^{(22)}$.

The vertical distribution of the ${ }^{226} \mathrm{Ra}$ activity concentration varied between $0.3 \pm 0.1$ and $4.8 \pm 0.1 \mathrm{~Bq}$ $\mathrm{m}^{-3}$ in winter 2007 , from $0.1 \pm 0.1$ to $9.0 \pm 0.8$ $\mathrm{Bq} \mathrm{m}{ }^{-3}$ in summer 2008 , from $0.4 \pm 0.2$ to $8.2 \pm 0.8$ $\mathrm{Bq} \mathrm{m}^{-3}$ in autumn 2008 and from $0.4 \pm 0.1$ to $2.3 \pm 0.3 \mathrm{~Bq} \mathrm{~m}^{-3}$ in winter 2009 (Table 3 and Figure 2). These values are in the same level as those reported in the eastern Mediterranean (e.g. $0.1-1.6 \mathrm{~Bq} \mathrm{~m}^{-3}$ in the Aegean Sea ${ }^{(25)}, 1.4-1.8 \mathrm{~Bq}$ $\mathrm{m}^{-3}$ in the North Mediterranean ${ }^{(26)}$. Radium isotopes have been used to study the water mass pathways or to provide information on horizontal and vertical ocean mixing (i) on a large-scale using ${ }^{226} \mathrm{Ra}^{(27-34)}$ and (ii) at the mesoscale using the ${ }^{232} \mathrm{Th}$-series radionuclide $\left.{ }^{228} \mathrm{Ra}^{(11,} 35-42\right)$. The 

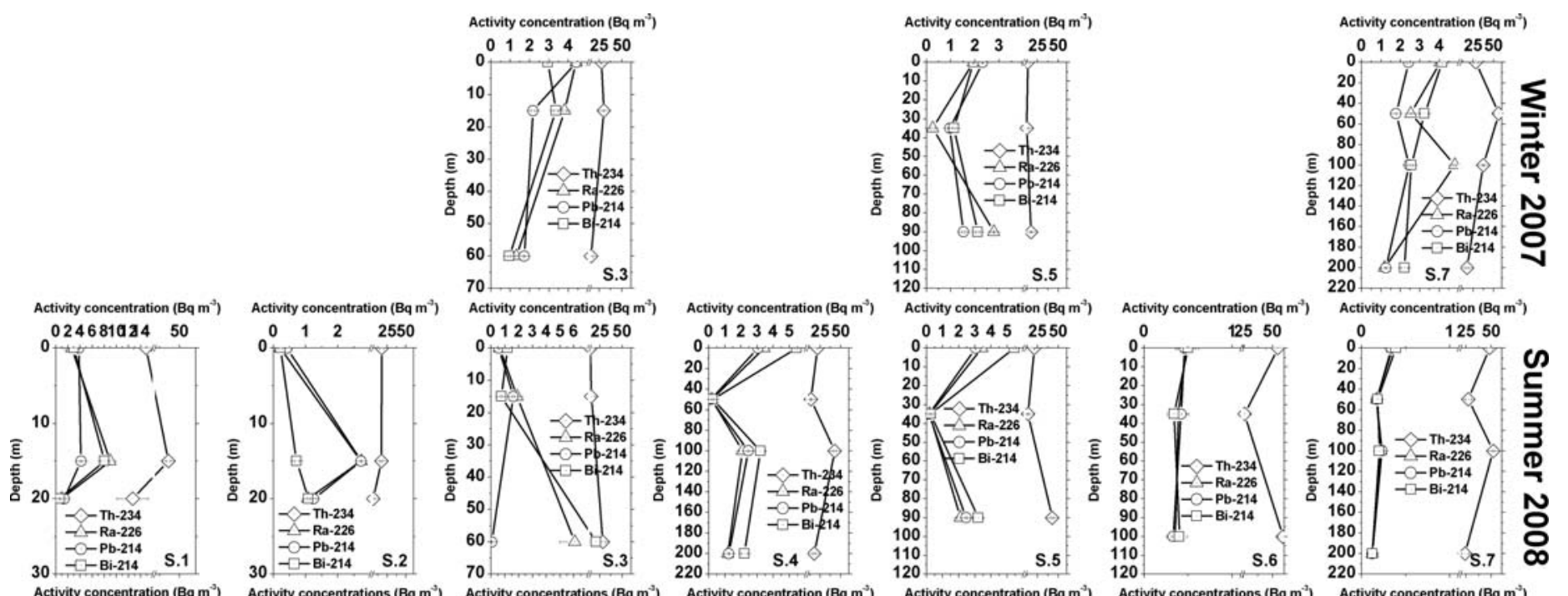

Activity concentration (Bq m
$012345 \quad 2550$
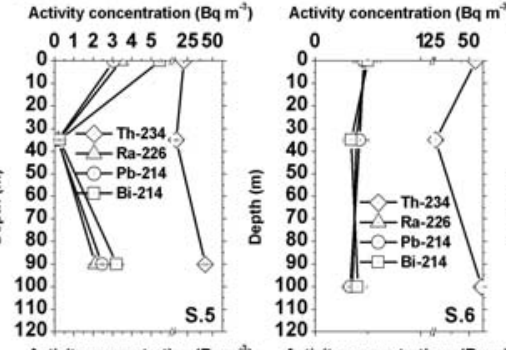

220
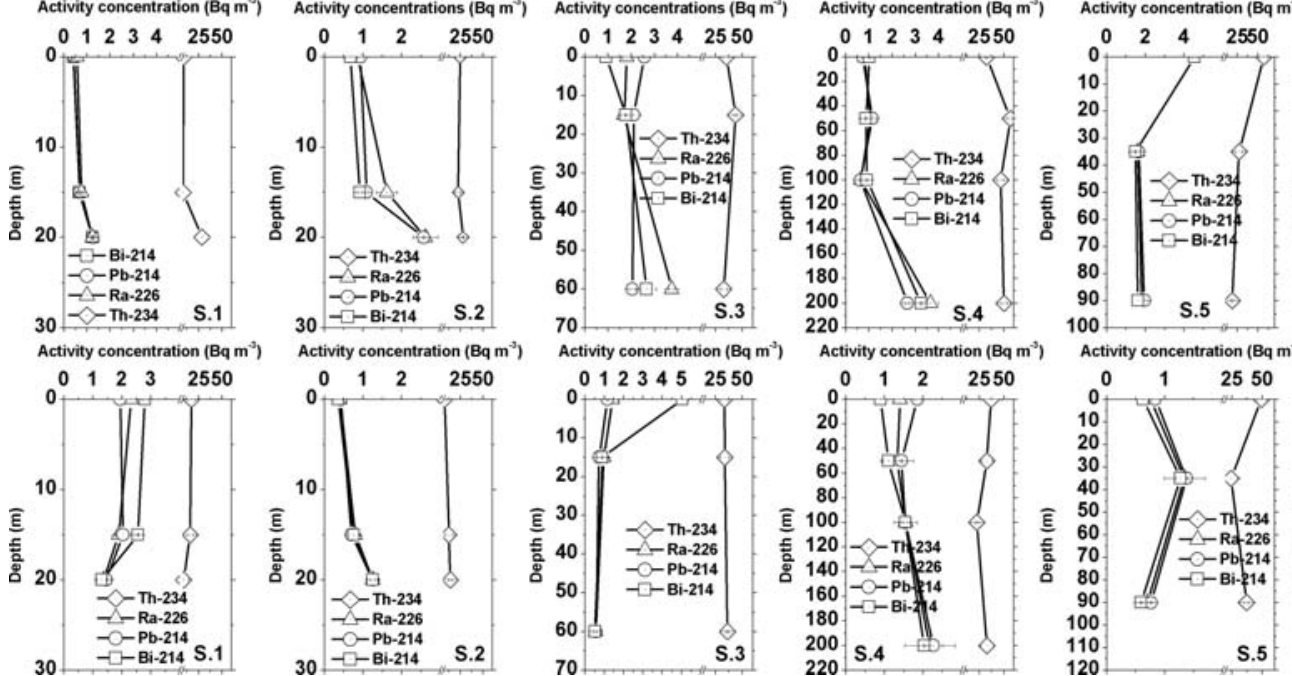

Activity concentrations $(\mathrm{Bq} \mathrm{m})$
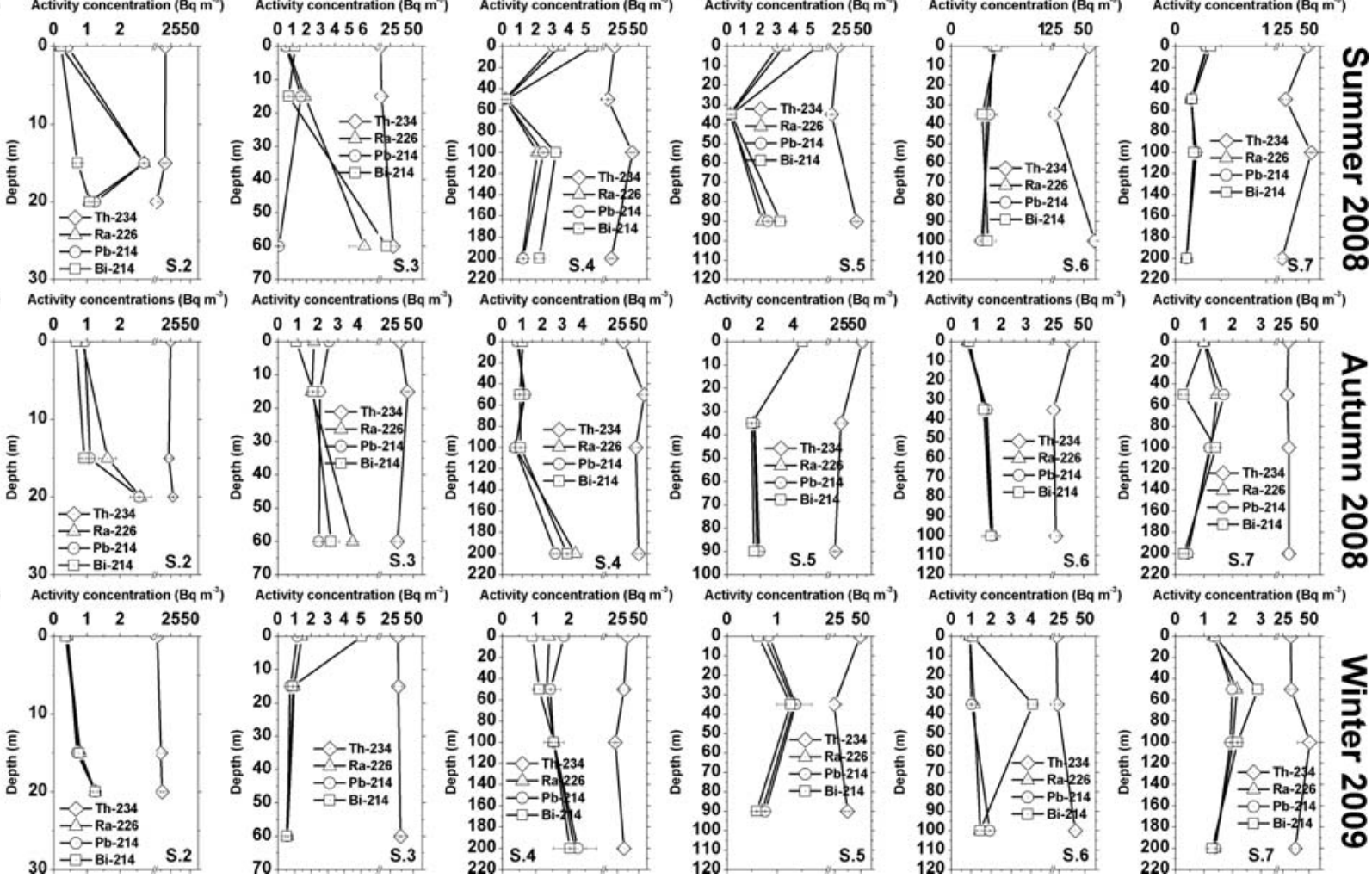

Figure 2. Vertical distribution of ${ }^{238} \mathrm{U}$-series radionuclides $\left({ }^{234} \mathrm{Th},{ }^{226} \mathrm{Ra},{ }^{214} \mathrm{~Pb}\right.$ and $\left.{ }^{214} \mathrm{Bi}\right)$ at the stations (S.1-S.7) of the Saronikos Gulf (Greece) during four different sampling campaigns. 


\section{N. EVANGELIOU ET AL.}

residence time of water masses could be estimated using ${ }^{228} \mathrm{Ra}^{(11)}$. $\mathrm{Ra}$ isotopes-including short-lived ${ }^{223} \mathrm{Ra}$ and ${ }^{224} \mathrm{Ra}$-have also been widely used to trace a submarine groundwater discharge $(\mathrm{SGD})^{(43-46)}$. In particular, they are used to assess groundwater discharge rates in coastal environments $^{(43,44,47)}$. However, radium concentrations were found low in the present work and, therefore, no similar processes can be quantified. Moreover, no impact from the phosphate fertiliser plant of Drapetsona can be recorded at the eastern section of the Saronikos Gulf or the Elefsis Bay.

With regard to ${ }^{222} \mathrm{Rn}$ daughters $\left({ }^{214} \mathrm{~Pb},{ }^{214} \mathrm{Bi}\right)$, their activity concentrations (corresponding to ${ }^{222} \mathrm{Rn}$ activity concentrations) were measured in a range from $0.9 \pm 0.1$ to $4.2 \pm 0.3 \mathrm{~Bq} \mathrm{~m}^{-3}$ in winter 2007, depending on the sampling depth and the topography, between $0.1 \pm 0.1$ and $20.2 \pm 5.4 \mathrm{~Bq} \mathrm{~m}^{-3}$ in summer 2008, between $0.3 \pm 0.1$ and $6.7 \pm 0.5 \mathrm{~Bq}$ $\mathrm{m}^{-3}$ in autumn 2008 and from $0.4 \pm 0.1$ to $5.0 \pm 0.3$ $\mathrm{Bq} \mathrm{m} \mathrm{m}^{-3}$ in winter 2009 (Table 3 and Figure 2). Although fluctuations were observed according to the depth, the average values of ${ }^{222} \mathrm{Rn}$ daughters' activity concentrations $\left({ }^{214} \mathrm{~Pb},{ }^{214} \mathrm{Bi}\right)$ were found almost equal, providing evidence that radioactive equilibrium between them has been obtained as expected. The present values are considered extremely low, although the available data refer to activity concentrations in areas of elevated artificial radioactivity (e.g. $>550 \mathrm{~Bq} \mathrm{~m}^{-3}$ in the south Ionian $\mathrm{Sea}^{(48)}$ and $100-3700 \mathrm{~Bq} \mathrm{~m}^{-3}$ in Sicily ${ }^{(49)}$ ).

\section{${ }^{232} \mathrm{Th}$-series radionuclides $\left({ }^{228} \mathrm{Ra}\right.$ and $\left.{ }^{228} \mathrm{Th}\right)$ \\ ${ }^{232} \mathrm{Th}$-series radionuclides, ${ }^{228} \mathrm{Ra}\left({ }^{228} \mathrm{Ac}\right)$ and ${ }^{228} \mathrm{Th}$} were quantitatively determined as well, together with ${ }^{232} \mathrm{Th}$ and the results are shown in Table 4. To obtain a more detailed view of the vertical distribution of the ${ }^{232} \mathrm{Th}$-series radionuclides, the respective vertical profiles are illustrated in Figure $3 .{ }^{228} \mathrm{Ra}$ was determined by the respective peaks of ${ }^{228} \mathrm{Ac}$ (at 338.4 and $911.1 \mathrm{keV}$; Table 2) after reaching secular equilibrium, whereas ${ }^{228} \mathrm{Th}$ by the respective peaks of ${ }^{212} \mathrm{~Pb}$ at $238.6 \mathrm{keV}$ and ${ }^{208} \mathrm{Tl}$ (where needed) at $583.1 \mathrm{keV}$ after secular equilibrium. ${ }^{232} \mathrm{Th}$ was calculated by the previously estimated daughter radionuclides ${ }^{228} \mathrm{Ra}$ and ${ }^{228} \mathrm{Th}$.

Activity concentrations of ${ }^{232} \mathrm{Th}$ ranged between $0.2 \pm 0.1$ and $3.6 \pm 0.2 \mathrm{~Bq} \mathrm{~m}^{-3}$ in winter 2007, between $0.1 \pm 0.1$ and $2.4 \pm 0.2 \mathrm{~Bq} \mathrm{~m}^{-3}$ in summer 2008 , from $0.2 \pm 0.1$ to $5.3 \pm 0.9 \mathrm{~Bq} \mathrm{~m}^{-3}$ in autumn 2008 and from $0.4 \pm 0.1$ to $1.8 \pm 0.3 \mathrm{~Bq} \mathrm{~m}^{-3}$ in winter 2009 depending on the sampling depth (Table 4, Figure 3). The maximum values were determined in the autumn period. This is an evidence of the evaporation of seawater during summer stratification resulting in dense surface waters that gradually sink to the bottom breaking down the
Table 4. The ${ }^{232} \mathrm{Th}$ series radionuclides ${ }^{228} \mathrm{Ra},{ }^{228} \mathrm{Th}$ and ${ }^{232} \mathrm{Th}$ in the seawater column of the stations in the Saronikos Gulf during the different sampling campaigns.

\begin{tabular}{|c|c|c|c|}
\hline & $\begin{array}{c}{ }^{232} \mathrm{Th} \\
\left(\mathrm{Bq} \mathrm{m}{ }^{-3}\right)\end{array}$ & $\left({ }^{228} \mathrm{Ra}\right.$ & $\left({ }^{28} \mathrm{Th}^{-3}\right)$ \\
\hline \multicolumn{4}{|l|}{ Winter 2007} \\
\hline $\mathrm{S} .3(0 \mathrm{~m})$ & $0.8 \pm 0.1$ & $0.8 \pm 0.2$ & $0.04 \pm 0.02$ \\
\hline $\mathrm{S} .3(15 \mathrm{~m})$ & $1.7 \pm 0.3$ & $1.3 \pm 0.1$ & $0.02 \pm 0.01$ \\
\hline $\mathrm{S} .3(60 \mathrm{~m})$ & $2.2 \pm 0.4$ & $1.8 \pm 0.1$ & $0.04 \pm 0.02$ \\
\hline $\mathrm{S} .5(0 \mathrm{~m})$ & $3.6 \pm 0.2$ & $1.9 \pm 0.5$ & $0.04 \pm 0.02$ \\
\hline $\mathrm{S} .5(35 \mathrm{~m})$ & $0.5 \pm 0.1$ & $0.1 \pm 0.1$ & $0.01 \pm 0.01$ \\
\hline S.5 (95 m) & $0.6 \pm 0.1$ & $1.5 \pm 0.4$ & $0.18 \pm 0.02$ \\
\hline $\mathrm{S} .7(0 \mathrm{~m})$ & $1.8 \pm 0.1$ & $2.6 \pm 0.1$ & $0.02 \pm 0.01$ \\
\hline $\mathrm{S} .7(50 \mathrm{~m})$ & $0.2 \pm 0.1$ & $0.2 \pm 0.1$ & $0.09 \pm 0.02$ \\
\hline S.7 (100 m) & $1.1 \pm 0.3$ & $1.8 \pm 0.4$ & $0.04 \pm 0.02$ \\
\hline $\mathrm{S} .7(200 \mathrm{~m})$ & $0.7 \pm 0.1$ & $0.7 \pm 0.1$ & $0.02 \pm 0.01$ \\
\hline \multicolumn{4}{|l|}{ Summer 2008} \\
\hline $\mathrm{S} .1(0 \mathrm{~m})$ & $2.4 \pm 0.2$ & $4.7 \pm 0.2$ & $0.05 \pm 0.03$ \\
\hline $\mathrm{S} .1(15 \mathrm{~m})$ & $2.4 \pm 0.1$ & $5.0 \pm 0.2$ & $0.02 \pm 0.01$ \\
\hline S.1 (20 m) & $1.0 \pm 0.1$ & $2.1 \pm 0.1$ & $0.01 \pm 0.01$ \\
\hline $\mathrm{S} .2(0 \mathrm{~m})$ & $0.2 \pm 0.1$ & $0.3 \pm 0.1$ & $0.04 \pm 0.02$ \\
\hline S.2 $(15 \mathrm{~m})$ & $0.7 \pm 0.1$ & $1.4 \pm 0.1$ & $0.01 \pm 0.01$ \\
\hline S. $2(20 \mathrm{~m})$ & $1.4 \pm 0.1$ & $2.8 \pm 0.1$ & $0.01 \pm 0.01$ \\
\hline $\mathrm{S} .3(0 \mathrm{~m})$ & $0.8 \pm 0.1$ & $1.8 \pm 0.1$ & $0.04 \pm 0.02$ \\
\hline $\mathrm{S} .3(15 \mathrm{~m})$ & $0.7 \pm 0.1$ & $1.6 \pm 0.1$ & $0.04 \pm 0.02$ \\
\hline $\mathrm{S} .3(60 \mathrm{~m})$ & $0.1 \pm 0.1$ & $0.1 \pm 0.1$ & $0.04 \pm 0.02$ \\
\hline $\mathrm{S} .4(0 \mathrm{~m})$ & $0.1 \pm 0.1$ & $0.1 \pm 0.1$ & $0.04 \pm 0.02$ \\
\hline $\mathrm{S} .4(50 \mathrm{~m})$ & $0.1 \pm 0.1$ & $0.1 \pm 0.1$ & $0.02 \pm 0.01$ \\
\hline $\mathrm{S} .4(100 \mathrm{~m})$ & $0.1 \pm 0.1$ & $0.2 \pm 0.1$ & $0.01 \pm 0.01$ \\
\hline $\mathrm{S} .4(200 \mathrm{~m})$ & $0.2 \pm 0.1$ & $0.4 \pm 0.1$ & $0.01 \pm 0.01$ \\
\hline $\mathrm{S} .5(0 \mathrm{~m})$ & $0.6 \pm 0.1$ & $1.3 \pm 0.3$ & $0.01 \pm 0.01$ \\
\hline $\mathrm{S} .5(35 \mathrm{~m})$ & $0.2 \pm 0.1$ & $0.3 \pm 0.1$ & $0.01 \pm 0.01$ \\
\hline $\mathrm{S} .5(95 \mathrm{~m})$ & $0.9 \pm 0.1$ & $2.9 \pm 0.1$ & $0.05 \pm 0.03$ \\
\hline $\mathrm{S} .6(0 \mathrm{~m})$ & $0.2 \pm 0.1$ & $0.4 \pm 0.1$ & $0.16 \pm 0.01$ \\
\hline S.6 (35 m) & $0.2 \pm 0.1$ & $0.3 \pm 0.1$ & $0.04 \pm 0.02$ \\
\hline S.6 $(100 \mathrm{~m})$ & $0.2 \pm 0.1$ & $0.4 \pm 0.1$ & $0.09 \pm 0.02$ \\
\hline $\mathrm{S} .7(0 \mathrm{~m})$ & $0.2 \pm 0.1$ & $0.3 \pm 0.1$ & $0.25 \pm 0.01$ \\
\hline $\mathrm{S} .7(50 \mathrm{~m})$ & $0.1 \pm 0.1$ & $0.2 \pm 0.1$ & $0.09 \pm 0.02$ \\
\hline S.7 $(100 \mathrm{~m})$ & $0.2 \pm 0.1$ & $0.2 \pm 0.1$ & $0.01 \pm 0.01$ \\
\hline S.7 $(200 \mathrm{~m})$ & $0.1 \pm 0.1$ & $0.2 \pm 0.1$ & $0.02 \pm 0.01$ \\
\hline \multicolumn{4}{|l|}{ Autumn 2008} \\
\hline $\mathrm{S} .1(0 \mathrm{~m})$ & $0.4 \pm 0.1$ & $2.0 \pm 0.1$ & $0.05 \pm 0.01$ \\
\hline $\mathrm{S} .1(15 \mathrm{~m})$ & $0.6 \pm 0.1$ & $3.2 \pm 0.1$ & $0.04 \pm 0.02$ \\
\hline S. $1(20 \mathrm{~m})$ & $0.7 \pm 0.2$ & $6.6 \pm 1.1$ & $0.02 \pm 0.01$ \\
\hline $\mathrm{S} .2(0 \mathrm{~m})$ & $0.7 \pm 0.2$ & $0.8 \pm 0.1$ & $0.04 \pm 0.02$ \\
\hline S.2 $(15 \mathrm{~m})$ & $1.2 \pm 0.3$ & $1.3 \pm 0.1$ & $0.02 \pm 0.01$ \\
\hline S.2 $(20 \mathrm{~m})$ & $1.8 \pm 0.4$ & $2.2 \pm 0.2$ & $0.01 \pm 0.01$ \\
\hline $\mathrm{S} .3(0 \mathrm{~m})$ & $1.1 \pm 0.4$ & $4.3 \pm 0.6$ & $0.01 \pm 0.01$ \\
\hline $\mathrm{S} .3(15 \mathrm{~m})$ & $1.0 \pm 0.1$ & $1.8 \pm 0.2$ & $0.05 \pm 0.02$ \\
\hline $\mathrm{S} .3(60 \mathrm{~m})$ & $3.0 \pm 0.7$ & $2.0 \pm 0.1$ & $0.04 \pm 0.02$ \\
\hline $\mathrm{S} .4(0 \mathrm{~m})$ & $0.5 \pm 0.1$ & $0.5 \pm 0.1$ & $0.02 \pm 0.01$ \\
\hline $\mathrm{S} .4(50 \mathrm{~m})$ & $0.7 \pm 0.1$ & $0.7 \pm 0.3$ & $0.05 \pm 0.01$ \\
\hline $\mathrm{S} .4(100 \mathrm{~m})$ & $0.4 \pm 0.1$ & $1.5 \pm 0.5$ & $0.05 \pm 0.02$ \\
\hline $\mathrm{S} .4(200 \mathrm{~m})$ & $1.6 \pm 0.3$ & $4.4 \pm 0.3$ & $0.01 \pm 0.01$ \\
\hline $\mathrm{S} .5(0 \mathrm{~m})$ & $5.3 \pm 0.9$ & $0.9 \pm 0.1$ & $0.05 \pm 0.01$ \\
\hline $\mathrm{S} .5(35 \mathrm{~m})$ & $1.2 \pm 0.4$ & $1.0 \pm 0.3$ & $0.04 \pm 0.02$ \\
\hline S.5 (95 m) & $1.5 \pm 0.1$ & $1.1 \pm 0.1$ & $0.04 \pm 0.02$ \\
\hline S. $6(0 \mathrm{~m})$ & $0.2 \pm 0.1$ & $2.6 \pm 0.8$ & $0.08 \pm 0.02$ \\
\hline S.6 (35 m) & $1.1 \pm 0.1$ & $0.8 \pm 0.2$ & $0.05 \pm 0.02$ \\
\hline S.6 $(100 \mathrm{~m})$ & $1.3 \pm 0.0$ & $0.5 \pm 0.1$ & $0.04 \pm 0.02$ \\
\hline
\end{tabular}

Continued 
Table 4. Continued

\begin{tabular}{|c|c|c|c|}
\hline & $\begin{array}{c}\left.{ }^{232} \mathrm{Th}^{-3}\right)\end{array}$ & $\left(\begin{array}{c}{ }^{228} \\
\left.{ }^{\mathrm{Bq}} \mathrm{m}^{-3}\right)\end{array}\right.$ & $\left({ }^{28} \mathrm{Th}^{-3}\right)$ \\
\hline $\mathrm{S} .7(0 \mathrm{~m})$ & $0.6 \pm 0.1$ & $0.6 \pm 0.1$ & $0.05 \pm 0.02$ \\
\hline S.7 (50 m) & $0.3 \pm 0.1$ & $2.9 \pm 0.1$ & $0.05 \pm 0.02$ \\
\hline $\mathrm{S} .7(100 \mathrm{~m})$ & $0.5 \pm 0.1$ & $0.3 \pm 0.1$ & $0.01 \pm 0.01$ \\
\hline S.7 $(200 \mathrm{~m})$ & $2.2 \pm 0.3$ & $1.6 \pm 0.1$ & $0.01 \pm 0.01$ \\
\hline \multicolumn{4}{|l|}{ Winter 2009} \\
\hline $\mathrm{S} .1(0 \mathrm{~m})$ & $1.2 \pm 0.3$ & $2.0 \pm 0.2$ & $0.13 \pm 0.02$ \\
\hline $\mathrm{S} .1(15 \mathrm{~m})$ & $0.9 \pm 0.1$ & $1.8 \pm 0.6$ & $0.05 \pm 0.02$ \\
\hline $\mathrm{S} .1(20 \mathrm{~m})$ & $0.5 \pm 0.1$ & $1.6 \pm 0.1$ & $0.01 \pm 0.01$ \\
\hline $\mathrm{S} .2(0 \mathrm{~m})$ & $0.4 \pm 0.1$ & $2.7 \pm 0.1$ & $0.09 \pm 0.02$ \\
\hline $\mathrm{S} .2(15 \mathrm{~m})$ & $1.8 \pm 0.1$ & $2.5 \pm 0.1$ & $0.04 \pm 0.02$ \\
\hline $\mathrm{S} .2(20 \mathrm{~m})$ & $1.0 \pm 0.3$ & $2.4 \pm 0.1$ & $0.01 \pm 0.01$ \\
\hline $\mathrm{S} .3(0 \mathrm{~m})$ & $1.3 \pm 0.1$ & $0.8 \pm 0.1$ & $0.01 \pm 0.01$ \\
\hline $\mathrm{S} .3(15 \mathrm{~m})$ & $0.7 \pm 0.3$ & $0.9 \pm 0.2$ & $0.01 \pm 0.01$ \\
\hline $\mathrm{S} .3(60 \mathrm{~m})$ & $0.4 \pm 0.1$ & $1.0 \pm 0.1$ & $0.01 \pm 0.01$ \\
\hline $\mathrm{S} .4(0 \mathrm{~m})$ & $1.0 \pm 0.3$ & $1.0 \pm 0.2$ & $0.08 \pm 0.03$ \\
\hline $\mathrm{S} .4(50 \mathrm{~m})$ & $1.2 \pm 0.1$ & $0.9 \pm 0.2$ & $0.04 \pm 0.02$ \\
\hline $\mathrm{S} .4(100 \mathrm{~m})$ & $1.3 \pm 0.1$ & $2.3 \pm 0.1$ & $0.04 \pm 0.02$ \\
\hline $\mathrm{S} .4(200 \mathrm{~m})$ & $1.8 \pm 0.3$ & $1.0 \pm 0.4$ & $0.01 \pm 0.01$ \\
\hline $\mathrm{S} .5(0 \mathrm{~m})$ & $0.4 \pm 0.1$ & $0.9 \pm 0.2$ & $0.08 \pm 0.02$ \\
\hline S. $5(35 \mathrm{~m})$ & $1.2 \pm 0.4$ & $0.7 \pm 0.2$ & $0.04 \pm 0.02$ \\
\hline $\mathrm{S} .5(95 \mathrm{~m})$ & $0.6 \pm 0.2$ & $1.1 \pm 0.2$ & $0.05 \pm 0.02$ \\
\hline S.6 $(0 \mathrm{~m})$ & $0.8 \pm 0.1$ & $1.7 \pm 0.1$ & $0.04 \pm 0.02$ \\
\hline S.6 (35 m) & $0.8 \pm 0.1$ & $1.0 \pm 0.2$ & $0.04 \pm 0.02$ \\
\hline $\mathrm{S} .6(100 \mathrm{~m})$ & $0.6 \pm 0.1$ & $1.0 \pm 0.2$ & $0.05 \pm 0.02$ \\
\hline $\mathrm{S} .7(0 \mathrm{~m})$ & $1.0 \pm 0.1$ & $0.6 \pm 0.2$ & $0.01 \pm 0.01$ \\
\hline $\mathrm{S} .7(50 \mathrm{~m})$ & $1.3 \pm 0.3$ & $2.1 \pm 0.3$ & $0.04 \pm 0.02$ \\
\hline S.7 $(100 \mathrm{~m})$ & $1.3 \pm 0.1$ & $1.1 \pm 0.1$ & $0.04 \pm 0.02$ \\
\hline S.7 (200 m) & $1.1 \pm 0.1$ & $1.0 \pm 0.3$ & $0.01 \pm 0.01$ \\
\hline
\end{tabular}

The final uncertainties were calculated by propagation.

thermocline layer ${ }^{(50)}$. The present values are up to one order of magnitude larger than those reported in the eastern Mediterranean region ${ }^{(25)}$.

Regarding ${ }^{228} \mathrm{Th}$, its activity concentration varied between $0.01 \pm 0.01$ and $0.18 \pm 0.02 \mathrm{~Bq} \mathrm{~m}^{-3}$ in winter 2007 , between $0.01 \pm 0.01$ to $0.25 \pm 0.01 \mathrm{~Bq}$ $\mathrm{m}^{-3}$ in summer 2008, between $0.01 \pm 0.01$ to $0.08 \pm 0.02 \mathrm{~Bq} \mathrm{~m}^{-3}$ in autumn 2008 and between $0.01 \pm 0.01$ to $0.13 \pm 0.02 \mathrm{~Bq} \mathrm{~m}^{-3}$ in winter 2009 (Table 4, Figure 3). These values are within the levels of the limit of detection $=0.01 \mathrm{~Bq} \mathrm{~m}^{-3}$ presenting a radiologically insignificant concentration.

Finally, ${ }^{228} \mathrm{Ra}$ activity concentrations fluctuated from $0.1 \pm 0.1$ to $2.6 \pm 0.1 \mathrm{~Bq} \mathrm{~m}^{-3}$ in winter 2007 , from $0.1 \pm 0.1$ to $5.0 \pm 0.2 \mathrm{~Bq} \mathrm{~m}^{-3}$ in summer 2008, from $0.3 \pm 0.1$ to $6.6 \pm 1.1 \mathrm{~Bq} \mathrm{~m}^{-3}$ in autumn 2008 and from $0.6 \pm 0.2$ to $2.7 \pm 0.1 \mathrm{~Bq} \mathrm{~m}^{-3}$ in winter 2009 (Table 4, Figure 3). The values observed are one order of magnitude lower than those reported in the western Mediterranean $\left(0.05-0.18 \mathrm{~Bq} \mathrm{~m}^{-3(51)}\right)$. However, ${ }^{228} \mathrm{Ra}$ has been found to increase in areas of elevated natural radioactivity of the eastern Mediterranean (e.g. $100-1100 \mathrm{~Bq} \mathrm{~m}^{-3(25,52,53)}$ ). It has been previously mentioned that $\mathrm{Ra}$ isotopes can be used to trace processes related to SGDs. However, the absence of fresh water discharges in the Saronikos Gulf limits the use of Ra isotopes as tracers. On the other hand, a preliminary approach can be performed for the fresh water discharges from the WWTP of Psitalia. These discharges comprise a simulated artificial groundwater system, although the origin of the outflowing waters may not be only terrigenous (and contain Ra isotopes), but also domestic.

The approach ${ }^{(54,55)}$ based on a box model is presented here using ${ }^{224} \mathrm{Ra}$ and ${ }^{228} \mathrm{Ra}$. If a system is at steady-state conditions, the addition and loss of Ra are equal. Additions of $\mathrm{Ra}$ are composed of fluxes from sediments, rivers and submarine waters, whereas losses by mixing with open ocean waters and physical decay. The balance equation is

$$
F^{224} \mathrm{Ra}=I^{224} \mathrm{Ra}\left(\lambda_{224}+\frac{1}{T_{\mathrm{w}}}\right)
$$

where $F^{224} \mathrm{Ra}$ is the net flux of the ${ }^{224} \mathrm{Ra}$ in the system $\left(\mathrm{Bq} \mathrm{m}{ }^{-2} \mathrm{~d}^{-1}\right), I^{224} \mathrm{Ra}$ is the inventory of the ${ }^{224} \mathrm{Ra}$ in the water column $\left(\mathrm{Bq} \mathrm{m}{ }^{-2}\right), \lambda_{224}$ is the decay constant of ${ }^{224} \mathrm{Ra}\left(\mathrm{d}^{-1}\right)$ and $T_{\mathrm{w}}$ is the residence time of ${ }^{224} \mathrm{Ra}(\mathrm{d})$. A similar equation can be written for ${ }^{228} \mathrm{Ra}$ as well. However, given that the half-life of ${ }^{228} \mathrm{Ra}$ is $5.8 \mathrm{y}$, the decay term can be assumed negligible. By dividing these equations, the flushing time, or residence time $\left(T_{\mathrm{w}}\right)$ can be determined:

$$
T_{\mathrm{w}}=\frac{\left[F\left({ }^{224} \mathrm{Ra} /{ }^{228} \mathrm{Ra}\right)-I\left({ }^{224} \mathrm{Ra} /{ }^{228} \mathrm{Ra}\right)\right]}{I\left({ }^{224} \mathrm{Ra} /{ }^{228} \mathrm{Ra}\right) \lambda_{224}}
$$

where $F\left({ }^{224} \mathrm{Ra} /{ }^{228} \mathrm{Ra}\right)$ is the activity ratio of the two isotopes near the groundwater discharge (flux) and $I\left({ }^{224} \mathrm{Ra} /{ }^{228} \mathrm{Ra}\right)$ is the activity ratio in the coastal mixing zone.

This model describes the residence time of the outflowing submarine groundwater before its mixing with the open seawater. The model demands accurate knowledge of the Ra fluxes by in situ measurements. As mentioned, this method is based on the experimental determination of the activity ratios of ${ }^{228} \mathrm{Ra}$ and ${ }^{224} \mathrm{Ra}$, isotopes of the same decay chain of ${ }^{232} \mathrm{Th}$. The activities of the isotopes are measured (a) at the outflow of the submarine groundwater flux and (b) at the area where the isotopes are concentrated after the outflow. In the present work, the outflow was at a depth of $60 \mathrm{~m}$ station S.3 (which is adjacent to the pipelines of the WWTP), whereas the authors supposed that the isotopes are concentrated near the thermocline (at a depth of $15 \mathrm{~m}$ ).

Since the direct determination of these radionuclides is unlikely using gamma spectrometry, an indirect method can be applied by estimating the 

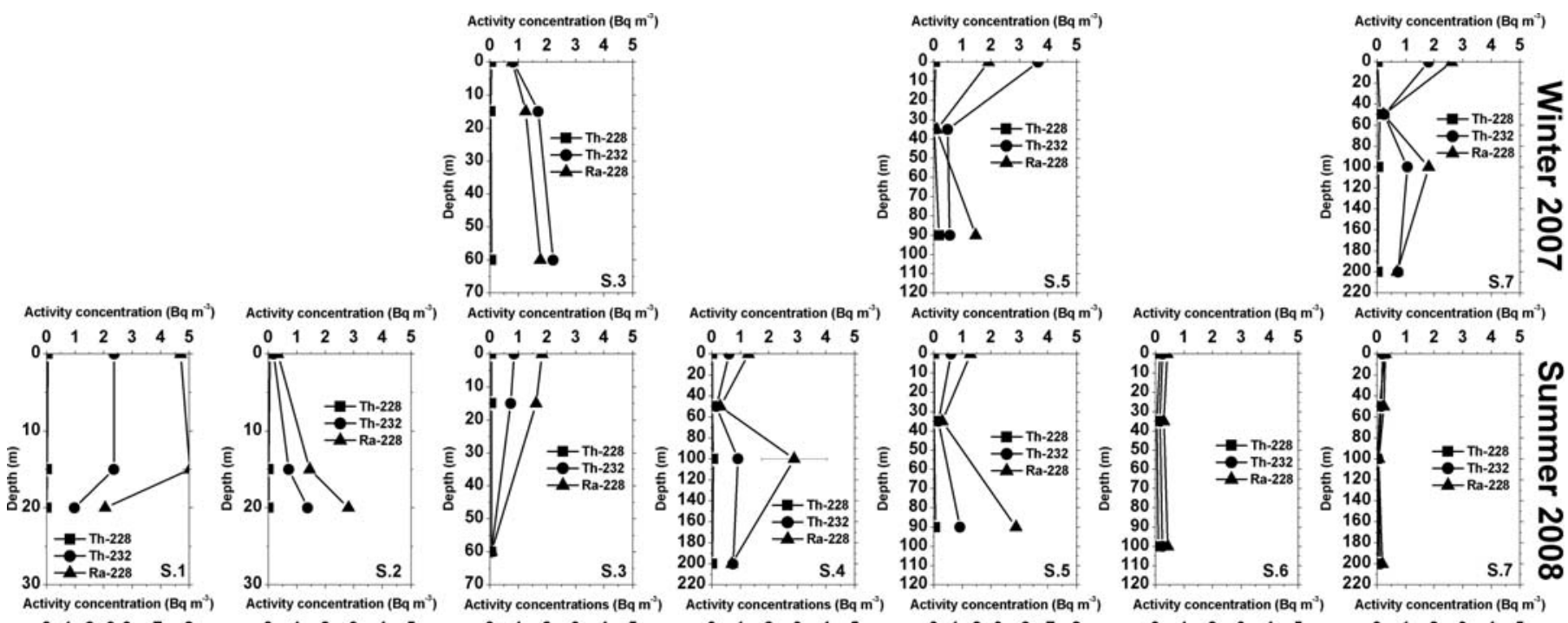

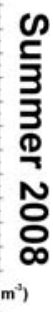
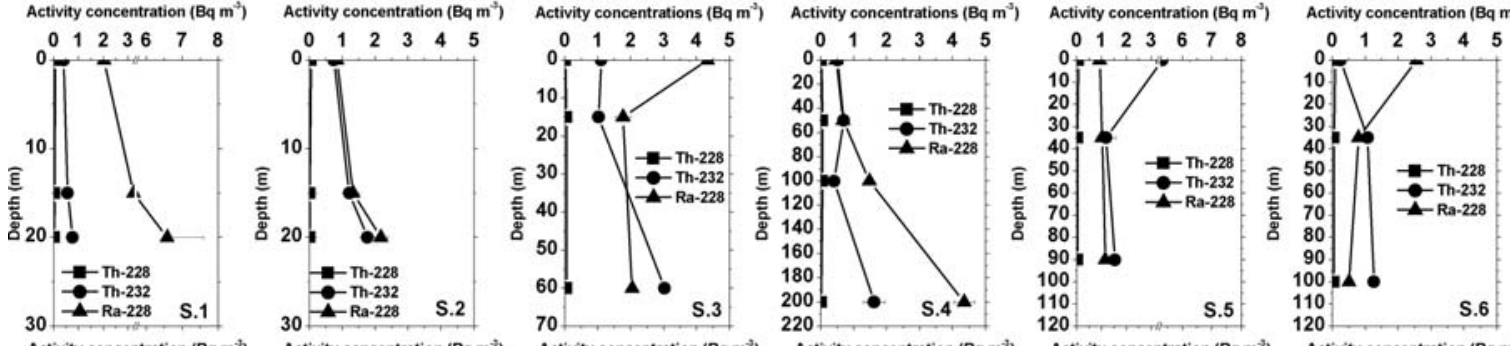

Activity concentration (Bq m
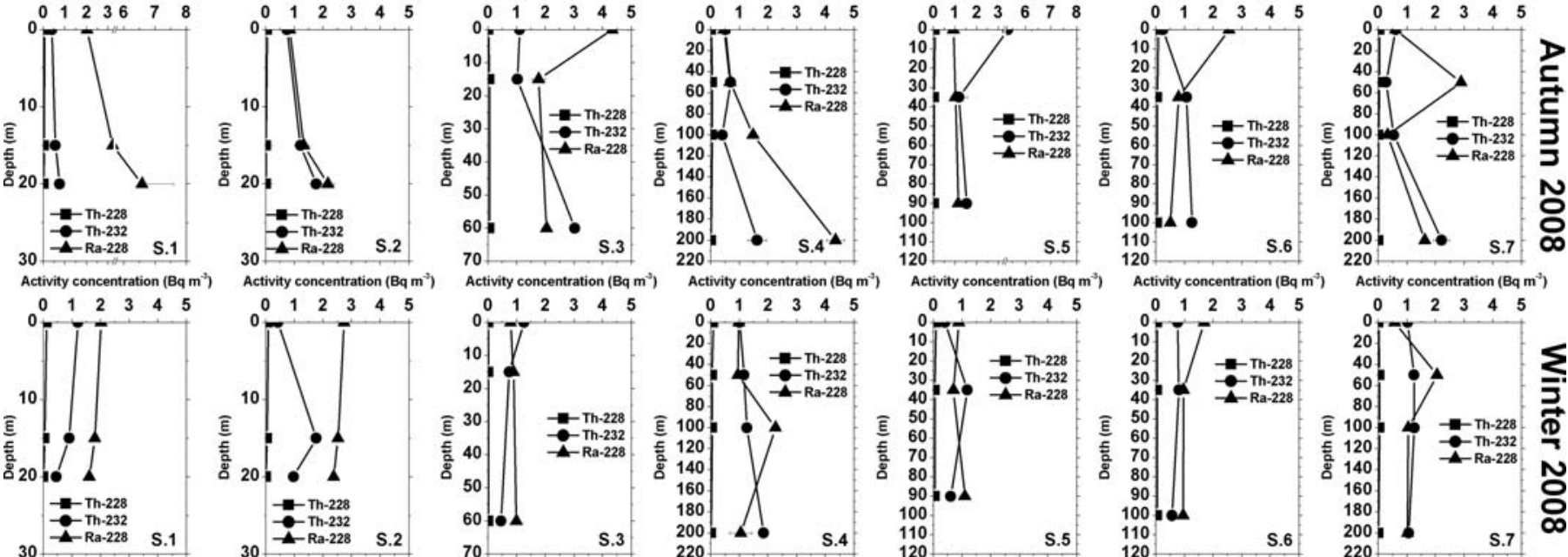

Figure 3. Vertical distribution of the ${ }^{232} \mathrm{Th}$-series radionuclides $\left({ }^{228} \mathrm{Ra}\right.$ and $\left.{ }^{228} \mathrm{Th}\right)$ at the stations (S.1-S.7) of the Saronikos Gulf (Greece) during four different sampling campaigns. 
DISTRIBUTION OF LOW-LEVEL NATURAL RADIOACTIVITY

Table 5. Estimated flushing times $\left(T_{w}\right)$ of the fresh water discharges after treatment at the WWTP of Psitalia.

\begin{tabular}{|c|c|c|c|c|c|}
\hline & \multicolumn{2}{|c|}{ S. $360 \mathrm{~m}(\mathrm{~F})$} & \multicolumn{2}{|c|}{ S. $315 \mathrm{~m}(\mathrm{I})$} & \multirow[b]{2}{*}{ Flushing time $T_{\mathrm{w}}(\mathrm{d})$} \\
\hline & ${ }^{208} \mathrm{Tl}$ (cpd) & ${ }^{228} \mathrm{Ac}(\mathrm{cpd})$ & ${ }^{208} \mathrm{Tl}$ (cpd) & ${ }^{228} \mathrm{Ac}(\mathrm{cpd})$ & \\
\hline Winter 2007 & $164 \pm 65$ & $311 \pm 89$ & $86 \pm 36$ & $389 \pm 92$ & $7.3 \pm 3.4$ \\
\hline Summer 2008 & $135 \pm 65$ & $300 \pm 97$ & $79 \pm 45$ & $357 \pm 99$ & $5.5 \pm 2.7$ \\
\hline Autumn 2008 & $189 \pm 78$ & $259 \pm 87$ & $62 \pm 33$ & $337 \pm 95$ & $15.7 \pm 7.6$ \\
\hline Winter 2009 & $156 \pm 56$ & $299 \pm 91$ & $71 \pm 35$ & $356 \pm 93$ & $8.6 \pm 4.5$ \\
\hline
\end{tabular}

The respective radionuclides were measured at the outflow of the pipeline (at $60 \mathrm{~m}$ of S.3) and at the thermocline depth (at $15 \mathrm{~m}$ of S.3) and the results are given in counts per day (cpd). The uncertainties are $1 \sigma$ values derived from counting statistics and the uncertainties of the flushing times $\left(T_{\mathrm{w}}\right)$ were estimated by propagation.

activities of the daughter isotopes, after sealing the samples and obtaining radioactive equilibrium. For that reason, activity concentrations of ${ }^{228} \mathrm{Ac}$ (daughter of ${ }^{228} \mathrm{Ra}$ ) and ${ }^{208} \mathrm{Tl}$ (daughter of ${ }^{224} \mathrm{Ra}$ ) could be determined after sealing. According to the decay chain of ${ }^{232} \mathrm{Th},{ }^{212} \mathrm{Bi}$ is transmuted to ${ }^{208} \mathrm{Tl}(35.9 \%)$ and to ${ }^{212} \mathrm{Po}(64.1 \%)$. Therefore ${ }^{208} \mathrm{Tl}$ activity concentrations were also normalised using the proper branching ratios in the decay scheme, in order to compare its activity with those of ${ }^{228} \mathrm{Ra}\left({ }^{228} \mathrm{Ac}\right)$ and examine if a secular equilibrium has occurred. The last equation can be modified to

$$
T_{\mathrm{w}}=\frac{F\left(\left({ }^{208} \mathrm{Tl}\right)_{\mathrm{cpd}} /\left({ }^{228} \mathrm{Ac}\right)_{\mathrm{cpd}}\right)-I\left(\left({ }^{208} \mathrm{Tl}\right)_{\mathrm{cpd}} /\left({ }^{228} \mathrm{Ac}\right)_{\mathrm{cpd}}\right)}{I\left(\left({ }^{208} \mathrm{Tl}\right)_{\mathrm{cpd}} /\left({ }^{228} \mathrm{Ac}\right)_{\mathrm{cpd}}\right) \lambda_{224}}
$$

where $F$ and $I$ are the activity ratios of the isotopic tracers, respectively, near the outflow (flux) and in the mixing zone (inventory) in counts per day (cpd), and $\lambda_{224}$ is the decay constant of ${ }^{224} \mathrm{Ra}\left(=0.189 \mathrm{~d}^{-1}\right)$.

The results of these estimates are depicted in Table 5. It can be observed that the residence times (or flushing times) of the fresh waters ranged between $5.5 \pm 2.7$ and $15.7 \pm 7.6 \mathrm{~d}$ depending on the sampling season. These values correspond to the time needed for the fresh waters to be mixed with the saline waters of the open sea after their outflow. It was assumed here that the mixing is likely at the depth of thermocline $(15 \mathrm{~m})$ of the same vertical water column, where the discharge occurred (S.3). This assumption may impose an error on the estimates obtained here (e.g. mixing can be significant in other adjacent regions of S.3). However, efforts were made to estimate the flushing times, assuming that the mixing lied at the station S.5 (which is $\sim 8$ $\mathrm{km}$ next to S.3) but negative results arose. A notable point in these values is the high value obtained during autumn $(15.7 \pm 7.6 \mathrm{~d})$. This is probably attributed to the stratification that occurs during summer in the Saronikos Gulf, which inhibits the mobility of seawater along the water column ${ }^{(56)}$. It should be noted here that the use of $\mathrm{Ra}$ isotopes as tracers of SGDs of terrigenous and domestic origin is a rough estimation. Nevertheless, it comprises the upper limit of the time needed to flow before their mixing with the surrounding seawater.

\section{${ }^{40} \mathrm{~K}$ estimated by salinity and density}

The results of the calculated ${ }^{40} \mathrm{~K}$ are shown in Table 6, together with the parameters of the calculation, salinity (S), density (D), estimated mass concentrations of stable- $\mathrm{K}[\mathrm{C}(\mathrm{K})]$ and ${ }^{40} \mathrm{~K}\left[\mathrm{C}\left({ }^{40} \mathrm{~K}\right)\right]$. The activity concentrations of ${ }^{40} \mathrm{~K}$ varied between 11 287 and $13152 \mathrm{~Bq} \mathrm{~m}^{-3}$ in winter 2007, between 11 007 and $13273 \mathrm{~Bq} \mathrm{~m}^{-3}$ in summer 2008, between 12342 and $13465 \mathrm{~Bq} \mathrm{~m}^{-3}$ in autumn 2008 and from 13008 to $13439 \mathrm{~Bq} \mathrm{~m}^{-3}$ in winter 2009. The maximum concentrations were found in autumn and winter and they are attributed to the terrigenous nature of ${ }^{40} \mathrm{~K}$, which gathers at the coastal regions of the gulf after rainfall and soil erosion. Regarding previous measurements of ${ }^{40} \mathrm{~K}$ in the Mediterranean, they are of similar levels, e.g. $12000-13000 \mathrm{~Bq} \mathrm{~m}^{-3}$ in the Aegean $\mathrm{Sea}^{(20)}, 12230 \pm 120 \mathrm{~Bq} \mathrm{~m}^{-3}$ in the Saronikos Gulf ${ }^{(57)}, 910-9200 \mathrm{~Bq} \mathrm{~m}^{-3}$ in the southern Ionian $\mathrm{Sea}^{(48)}$ and $6000-13000 \mathrm{~Bq} \mathrm{~m}^{-3}$ in central Greece $^{(25)}$.

\section{Ratios of ${ }^{238} \mathrm{U}-{ }^{232} \mathrm{Th}$ and ${ }^{40} \mathrm{~K}-{ }^{232} \mathrm{Th}$}

The respective ratios of ${ }^{232} \mathrm{Th}$ and ${ }^{238} \mathrm{U}$ activity concentrations (Figure 4a) were calculated for all samples in order to assess if anthropogenic activities are present in the area. However, ${ }^{238} \mathrm{U}$ was calculated by its proportionality with salinity though there are some values influenced by salinity corresponding to the stations near the WWTP of Psitalia, where low-saline waters outflow. Moreover, compared with Th isotopes, it presents different chemical behaviour in seawater. For that reason, the ratio of its daughter products was calculated $\left({ }^{226} \mathrm{Ra} /{ }^{228} \mathrm{Ac}\right)$, 
Table 6. Salinity and density data by in situ measurements, calculated mass concentrations of stable- $K[C(K)]$ and ${ }^{40} K\left[C\left({ }^{40} K\right)\right]$ and activity concentrations of ${ }^{40} K\left[A\left({ }^{40} K\right)\right]$.

\begin{tabular}{|c|c|c|c|c|c|c|c|c|c|c|c|c|c|c|c|c|c|c|c|c|}
\hline \multirow[b]{2}{*}{ Station } & \multicolumn{5}{|c|}{ Winter 2007} & \multicolumn{5}{|c|}{ Summer 2008} & \multicolumn{5}{|c|}{ Autumn 2008} & \multicolumn{5}{|c|}{ Winter 2009} \\
\hline & $\mathrm{S}(\mathrm{psu})$ & $\underset{\left(\mathrm{kg} \mathrm{m}^{-3}\right)}{\mathrm{D}}$ & $\begin{array}{c}\mathrm{C}(\mathrm{K}) \\
\left(\mathrm{g} \mathrm{m}^{-3}\right)\end{array}$ & $\begin{array}{l}\mathrm{C}\left({ }^{40} \mathrm{~K}\right) \\
\left(\mathrm{g} \mathrm{m}^{-3}\right)\end{array}$ & $\begin{array}{c}\mathrm{A}\left({ }^{40} \mathrm{~K}\right) \\
\left.(\mathrm{Bq} \mathrm{m})^{-3}\right)\end{array}$ & $\mathrm{S}(\mathrm{psu})$ & $\underset{\left(\mathrm{kg} \mathrm{m}^{-3}\right)}{\mathrm{D}}$ & $\begin{array}{c}\mathrm{C}(\mathrm{K}) \\
\left(\mathrm{g} \mathrm{m}^{-3}\right)\end{array}$ & $\begin{array}{l}\mathrm{C}\left({ }^{40} \mathrm{~K}\right) \\
\left(\mathrm{g} \mathrm{m}^{-3}\right)\end{array}$ & $\begin{array}{l}\mathrm{A}\left({ }^{40} \mathrm{~K}\right) \\
\left(\mathrm{Bq} \mathrm{\textrm {m } ^ { - 3 }}\right)\end{array}$ & $\mathrm{S}(\mathrm{psu})$ & $\underset{\left(\mathrm{kg} \mathrm{m}^{-3}\right)}{\mathrm{D}}$ & $\begin{array}{c}\mathrm{C}(\mathrm{K}) \\
\left(\mathrm{g} \mathrm{m}^{-3}\right)\end{array}$ & $\begin{array}{l}\mathrm{C}\left({ }^{40} \mathrm{~K}\right) \\
\left(\mathrm{g} \mathrm{m}^{-3}\right)\end{array}$ & $\begin{array}{c}\mathrm{A}\left({ }^{40} \mathrm{~K}\right) \\
\left(\mathrm{Bq} \mathrm{\textrm {m } ^ { - 3 }}\right)\end{array}$ & S (psu) & $\underset{\left(\mathrm{kg} \mathrm{m}^{-3}\right)}{\mathrm{D}}$ & $\begin{array}{c}\mathrm{C}(\mathrm{K}) \\
\left(\mathrm{g} \mathrm{m}^{-3}\right)\end{array}$ & $\begin{array}{l}\mathrm{C}\left({ }^{40} \mathrm{~K}\right) \\
\left(\mathrm{g} \mathrm{m}^{-3}\right)\end{array}$ & $\begin{array}{c}\mathrm{A}\left({ }^{40} \mathrm{~K}\right) \\
\left.(\mathrm{Bq} \mathrm{m})^{-3}\right)\end{array}$ \\
\hline S.1 (0 m) & & & & & & 38.5 & 1025.49 & 434 & 0.051 & 13139 & 38.9 & 1027.47 & 440 & 0.051 & 13302 & 38.6 & 1029.04 & 437 & 0.051 & 13219 \\
\hline S.1 (15 m) & & & & & & 34.2 & 1024.65 & 386 & 0.045 & 11662 & 38.6 & 1027.19 & 436 & 0.051 & 13195 & 38.1 & 1028.48 & 431 & 0.050 & 13041 \\
\hline S.1 (20 m) & & & & & & 34.0 & 1024.62 & 383 & 0.045 & 11594 & 38.5 & 1027.22 & 435 & 0.051 & 13162 & 38.9 & 1029.25 & 440 & 0.052 & 13325 \\
\hline S.2 (0 m) & & & & & & 38.9 & 1025.23 & 439 & 0.051 & 13273 & 37.2 & 1025.28 & 420 & 0.049 & 12693 & 38.3 & 1028.85 & 433 & 0.051 & 13114 \\
\hline S. $2(15 \mathrm{~m})$ & & & & & & 34.1 & 1024.35 & 384 & 0.045 & 11625 & 36.2 & 1024.47 & 408 & 0.048 & 12342 & 38.0 & 1028.62 & 430 & 0.050 & 13008 \\
\hline $\mathrm{S} .2(20 \mathrm{~m})$ & & & & & & 33.9 & 1024.68 & 382 & 0.045 & 11560 & 37.0 & 1026.29 & 418 & 0.049 & 12637 & 38.5 & 1028.81 & 436 & 0.051 & 13182 \\
\hline S.3 $(0 \mathrm{~m})$ & 36.7 & 1027.37 & 415 & 0.049 & 12548 & 38.7 & 1026.00 & 437 & 0.051 & 13214 & 38.7 & 1027.01 & 437 & 0.051 & 13227 & 39.2 & 1029.03 & 444 & 0.052 & 13424 \\
\hline S. $3(15 \mathrm{~m})$ & 36.4 & 1027.17 & 411 & 0.048 & 12443 & 34.1 & 1024.62 & 384 & 0.045 & 11628 & 37.8 & 1026.18 & 427 & 0.050 & 12909 & 38.9 & 1028.91 & 440 & 0.052 & 13320 \\
\hline S.3 $(60 \mathrm{~m})$ & 33.1 & 1024.64 & 373 & 0.044 & 11287 & 32.3 & 1023.96 & 364 & 0.043 & 11007 & 36.2 & 1026.07 & 409 & 0.048 & 12361 & 38.5 & 1028.66 & 436 & 0.051 & 13180 \\
\hline $\mathrm{S} .4(0 \mathrm{~m})$ & & & & & & 38.2 & 1025.76 & 431 & 0.050 & 13040 & 38.4 & 1026.46 & 434 & 0.051 & 13118 & 38.6 & 1028.64 & 437 & 0.051 & 13214 \\
\hline S.4 $(50 \mathrm{~m})$ & & & & & & 33.9 & 1025.12 & 382 & 0.045 & 11565 & 38.2 & 1027.43 & 432 & 0.051 & 13062 & 38.8 & 1029.04 & 439 & 0.051 & 13288 \\
\hline $\mathrm{S} .4(100 \mathrm{~m})$ & & & & & & 33.7 & 1025.31 & 380 & 0.044 & 11499 & 38.3 & 1028.19 & 433 & 0.051 & 13106 & 39.1 & 1029.49 & 443 & 0.052 & 13396 \\
\hline S. $4(200 \mathrm{~m})$ & & & & & & 33.3 & 1025.53 & 376 & 0.044 & 11365 & 38.9 & 1029.24 & 440 & 0.052 & 13324 & 39.2 & 1030.12 & 444 & 0.052 & 13439 \\
\hline S.5 $(0 \mathrm{~m})$ & 38.1 & 1028.52 & 431 & 0.050 & 13041 & 38.3 & 1025.75 & 432 & 0.051 & 13074 & 38.0 & 1026.09 & 429 & 0.050 & 12976 & 39.1 & 1028.95 & 443 & 0.052 & 13389 \\
\hline S. $5(35 \mathrm{~m})$ & 37.9 & 1028.69 & 429 & 0.050 & 12975 & 34.5 & 1025.28 & 389 & 0.046 & 11772 & 38.8 & 1027.76 & 439 & 0.051 & 13271 & 39.0 & 1028.89 & 441 & 0.052 & 13354 \\
\hline S. $5(95 \mathrm{~m})$ & 38.4 & 1029.13 & 435 & 0.051 & 13152 & 32.8 & 1024.59 & 370 & 0.043 & 11184 & 38.5 & 1027.99 & 435 & 0.051 & 13171 & 39.0 & 1029.22 & 442 & 0.052 & 13358 \\
\hline S.6 (0 m) & & & & & & 38.1 & 1025.59 & 430 & 0.050 & 13004 & 38.5 & 1026.80 & 435 & 0.051 & 13156 & 38.5 & 1028.54 & 436 & 0.051 & 13178 \\
\hline S.6 $(35 \mathrm{~m})$ & & & & & & 35.0 & 1025.48 & 395 & 0.046 & 11945 & 39.0 & 1027.97 & 441 & 0.052 & 13342 & 38.8 & 1029.40 & 439 & 0.051 & 13292 \\
\hline S.6 $(100 \mathrm{~m})$ & & & & & & 34.2 & 1025.58 & 386 & 0.045 & 11673 & 37.9 & 1027.81 & 429 & 0.050 & 12964 & 39.1 & 1029.70 & 443 & 0.052 & 13399 \\
\hline S.7 $(0 \mathrm{~m})$ & 38.4 & 1028.73 & 435 & 0.051 & 13147 & 37.8 & 1025.46 & 426 & 0.050 & 12900 & 38.9 & 1027.07 & 440 & 0.051 & 13296 & 39.0 & 1028.95 & 441 & 0.052 & 13355 \\
\hline S.7 (50 m) & 38.1 & 1028.81 & 431 & 0.050 & 13045 & 34.1 & 1023.47 & 384 & 0.045 & 11615 & 38.6 & 1027.92 & 436 & 0.051 & 13205 & 39.1 & 1029.27 & 443 & 0.052 & 13393 \\
\hline S.7 $(100 \mathrm{~m})$ & 37.7 & 1028.75 & 427 & 0.050 & 12907 & 33.9 & 1024.01 & 382 & 0.045 & 11553 & 38.6 & 1028.42 & 437 & 0.051 & 13211 & 38.7 & 1029.23 & 438 & 0.051 & 13256 \\
\hline S.7 $(200 \mathrm{~m})$ & 36.5 & 1028.23 & 413 & 0.048 & 12490 & 33.7 & 1024.78 & 380 & 0.044 & 11493 & 39.3 & 1029.48 & 445 & 0.052 & 13465 & 39.0 & 1029.97 & 442 & 0.052 & 13368 \\
\hline
\end{tabular}



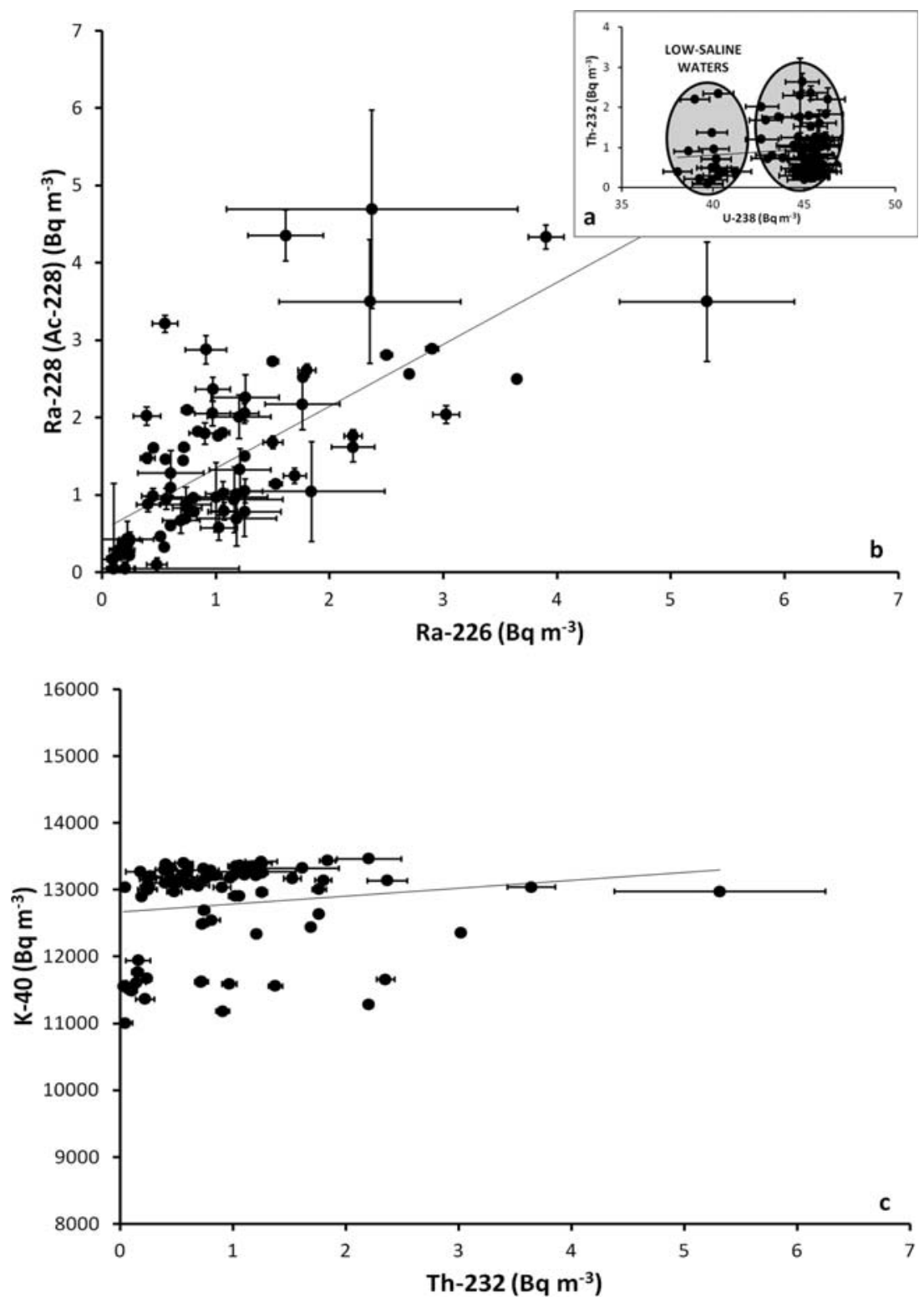

Figure 4. Correlation of (a) ${ }^{238} \mathrm{U}$ vs. ${ }^{232} \mathrm{Th}$, (b) ${ }^{226} \mathrm{Ra}$ vs. ${ }^{228} \mathrm{Ra}\left({ }^{228} \mathrm{Ac}\right)$ and (c) ${ }^{40} \mathrm{~K}$ vs. ${ }^{232} \mathrm{Th}$ activity concentrations.

assuming secular equilibrium, by a least square fitting (Figure $4 \mathrm{~b}$ ) and it was found to be 0.8 with a correlation coefficient of $R^{2}=0.59$. Considering that secular radioactive equilibrium exists between ${ }^{238} \mathrm{U}-{ }^{226} \mathrm{Ra}$ and ${ }^{232} \mathrm{Th}-{ }^{228} \mathrm{Ac}$, this value $\left({ }^{226} \mathrm{Ra} /{ }^{228} \mathrm{Ac}\right)$ was compared and found to be low ${ }^{(15,58)}$, indicating that the TENORM contribution from human activities could be considered negligible.
Similarly, correlations between ${ }^{40} \mathrm{~K}$ and ${ }^{232} \mathrm{Th}$ were also considered. The measurements of ${ }^{40} \mathrm{~K}$ and the ${ }^{232} \mathrm{Th}$ activity concentrations were linearly fitted and are illustrated in Figure $4 \mathrm{c}$ where a significant correlation was observed $\left(R^{2}=0.59\right)$. The results indicate that there is a positive correlation between the activities of ${ }^{40} \mathrm{~K}$ and ${ }^{232} \mathrm{Th}$. Thus, from the slope of the linear fitting the mean value of the ratio of ${ }^{40} \mathrm{~K} /{ }^{232} \mathrm{Th}$ was found to be equal to 125 . This value 


\section{N. EVANGELIOU ET AL.}

is at the lowest level compared with those reported in the eastern Mediterranean region (e.g. $49 \times 10^{4(25)}$ ) and much higher than those observed in surface sediments (e.g. 25 in the Ionian Sea ${ }^{(59)}$ ), as a result of the ionic presence of potassium in seawater in conjunction with a low affinity to bind particles.

\section{CONCLUSIONS}

The activity concentrations of the ${ }^{238} \mathrm{U}$-series and ${ }^{232} \mathrm{Th}$-series radionuclides and ${ }^{40} \mathrm{~K}$, as well, have been recorded as a baseline study of the present levels of natural radioactivity in a small embayment of the eastern Mediterranean Sea.

The activity concentrations of the ${ }^{238} \mathrm{U}$-series radionuclides were found comparable with or lower than those observed previously in Mediterranean regions, showing that the sampling location is not affected by TENORM activities although there was a fertilisers' plant on its eastern coast.

Relatively similar results were observed for ${ }^{232} \mathrm{Th}$ series radionuclides in the water column in comparison with the relevant literature for the Mediterranean Sea, although the ${ }^{232}$ Th activity concentrations calculated by its daughter products were up to one order of magnitude higher. The activity concentrations of ${ }^{228} \mathrm{Ra}$ are more than one order of magnitude lower than those of ${ }^{228} \mathrm{Th}$. On the other hand, the activity concentration of ${ }^{208} \mathrm{Tl}$ at the end of the decay chain seems to be almost in the same order as the activity concentration of ${ }^{228} \mathrm{Ac}$. So the activity concentration of ${ }^{228} \mathrm{Th}$ is extremely low when compared with its parent and daughter products. This is not surprising since it is clearly attributed to the nature of Th. As mentioned previously, Th is a particle-reactive element having strong affinity to get adsorbed onto marine particles. Marine particles are rapidly removed from the water column via sinking to the sediments (e.g. Buesseler et al. ${ }^{(60)}$ and many others on the same issue) and as a result, lack of equilibrium between the mother and daughter products in seawater is usually observed.

${ }^{40} \mathrm{~K}$ estimated by the salinity and density of seawater was found in the range of previous measurements of eastern Mediterranean areas (11 000-14 $000 \mathrm{~Bq} \mathrm{~m}^{-3}$ ).

A rough estimation of the residence times of fresh waters that outflow from the WWTP of Psitalia was attempted, assuming that the origin of the outfalling waters is exclusively terrigenous. An upper level of the time it takes for fresh waters to be mixed with seawater after their outflow was derived to be equal to $15.7 \pm 7.6 \mathrm{~d}$.

\section{SUPPLEMENTARY DATA}

Supplementary data are available at Radiation Protection Dosimetry.

\section{ACKNOWLEDGEMENTS}

The authors acknowledge the captain (Mr P. Katsakos) and the crew (Mr P. Marantidis) of the vessels 'Agios Efthymios' and 'Agios Efstratios' for their assistance. Finally, the authors appreciate Marine Environment Laboratory of IAEA in Monaco and Dr Iolanda Osvath, in personal, for borrowing a pair of McLane in situ samplers.

\section{FUNDING}

The present study was financially supported by NCSR 'Demokritos'/Institute of Nuclear Technology_Radiation Protection/Environmental Radioactivity Laboratory.

\section{REFERENCES}

1. Vera Tome, F., Blanco Rodriguez, M. P. and Lozano, J. C. Soil-to-plant transfer factors for natural radionuclides and stable elements in a Mediterranean area. J. Environ. Radioact. 65, 161-175 (2003).

2. UNSCEAR. Source and effects of ionizing radiation. United Nations Scientific Committee on the Effects of Atomic Radiation. United Nations (2000).

3. Chen, S. B., Zhu, Y. G. and Hu, Q. H. Soil to plant transfer of ${ }^{238} \mathrm{U},{ }^{226} \mathrm{Ra}$ and ${ }^{232} \mathrm{Th}$ on uranium miningimpacted soil from southeastern China. J. Environ. Radioact. 82, 223-236 (2005).

4. Amaral, R. S., Vasconcelos, W. E., Borges, E., Silveira, S. V. and Barbara, P. M. Intake of uranium and radium226 due to food crops consumption in the phosphate region of Pernambuco e Brazil. J. Environ. Radioact. 82, 383-393 (2005).

5. Voutsinou-Taliadouri, E. Metal pollution in the Saronikos Gulf. Mar. Poll. Bull. 12, 163-168 (1981).

6. IAEA. Naturally occurring radioactive material NORM. In: Proceedings of an International Symposium, Seville, Spain, 19-22 March 2007.

7. Stamatis, V., Seferlis, S., Kamenopoulou, V., Potiriadis, C., Koukouliou, V., Kehagia, K., Dagli, C., Georgiadis, S. and Camarinopoulos, L. Decommissioning a phosphoric acid production plant: a radiological protection case study. J. Environ. Radioact. 101, 1013-1023 (2010).

8. Simpson, W. R. Deep-sea in situ particle samplers (Using the Mark III Centrifugal Pump). Operating Manual. Challenger Oceanic Systems and Services (1997).

9. Bacon, M. P. and Anderson, R. F. Distribution of thorium isotopes between dissolved and particulate forms in the deep sea. J. Geophys. Res. 87, 2045-2056 (1982).

10. Hanfland, C. Radium-226 and radium-228 in the Atlantic sector of the Southern Ocean. Berichte zur Polar und Meeresforchung 431. Ph.D. thesis, Alfred Wegener Institute, Bremerhaven, Germany, pp. 135 (2002).

11. Rutgers van der Loeff, M. M. ${ }^{228} \mathrm{Ra}$ and ${ }^{228}$ Th in the Weddell Sea. In: The Polar Oceans and Their Role in Shaping the Global Environment: The Nansen Centennial Volume. Geophysical Monograph 85, 


\section{DISTRIBUTION OF LOW-LEVEL NATURAL RADIOACTIVITY}

Johannessen, O. M., Muench, R. D. and Overland, J. E. Eds. American Geophysical Union. pp. 177-186 (1994).

12. Rutgers van der Loeff, M. M. and Berger, G. W. Scavenging of ${ }^{230}$ Th and ${ }^{231} \mathrm{~Pa}$ near the Antarctic Polar Front in the South Atlantic. Deep-Sea Res. I 40, 339-357 (1993).

13. Mann, D. R., Surprenant, L. D. and Casso, S. A. In situ chemisorption of transuranics from seawater. Nucl. Instrum. Methods Phys. Res. Sect. A 223, 235-238 (1984).

14. Livingston, H. D. and Cochran, J. K. Determination of transuranic and thorium isotopes in ocean water: in solution and in filterable particles. J. Radioanal. Nucl. Chem. Artic. 115(2), 299-308 (1987)

15. Chen, J. H., Edwards, R. L. and Wasserburg, G. J. ${ }^{238} U,{ }^{234} U$ and ${ }^{232} T h$ in seawater. Earth Planet. Sci. Lett. 80, 241-251 (1986).

16. Carroll, J. and Moore, W. S. Uranium removal during low discharge in the Ganges-Brahmaputra mixing zone. Geochim. Cosmochim. Acta 57, 4987-4995 (1993).

17. Kersten, M., Thomsen, S., Priebsch, W. and GarbeSchoenberg, C. D. Scavenging and particle residence times determined from ${ }^{234} \mathrm{Th} /{ }^{238} \mathrm{U}$ disequilibria in the coastal waters of Mecklenburg Bay. Appl. Geochem. 13(3), 339-347 (1998).

18. McKee, B. A., DeMaster, D. J. and Nittrouer, C. A. Uranium geochemistry on the Amazon shelf-evidence for uranium release from bottom sediments. Geochim. Cosmochim. Acta 51, 2779-2786 (1987).

19. Delanghe, D., Bard, E. and Hamelin, B. New TIMS constraints on the uranium-238 and uranium-234 in seawaters from the main ocean basins and the Mediterranean Sea. Mar. Chem. 80, 79-93 (2002).

20. Tsabaris, C. and Ballas, D. On line gamma-ray spectrometry at open sea. Appl. Radiat. Isot. 62, 83-89 (2005).

21. Pates, J. M. and Muir, G. K. P. U-salinity relationships in the Mediterranean: implications for ${ }^{234} \mathrm{Th}^{.238} \mathrm{U}$ particle flux studies. Mar. Chem. 106, 530-545 (2007).

22. Speicher, E. A. et al. Particulate organic carbon export fluxes and size-fractionated $P O C{ }^{234}$ Th ratios in the Ligurian, Tyrrhenian and Aegean Seas. Deep-Sea Res. I 53, 1810-1830 (2006).

23. Buesseler, K. O., Cochran, J. K., Bacon, M. P., Livingston, H. D., Casso, S. A., Hirschberg, D., Hartman, M. C. and Fleer, A. P. Determination of thorium isotopes in seawater by non-destructive and radiochemical procedures. Deep-Sea Res. 39(7/8), 1103-1114 (1992).

24. Charette, M. A. and Moran, S. B. Rates of particle scavenging and particulate organic carbon export estimated using ${ }^{234}$ Th as a tracer in the subtropical and equatorial Atlantic Ocean. Deep-Sea Res. 46, 885-906 (1999).

25. Florou, H., Trabidou, G. and Nicolaou, G. An assessment of the external radiological impact in areas of Greece with elevated natural radioactivity. J. Environ. Radioact. 93(2), 74-83 (2007).

26. Masqué, P., Sanchez-Cabeza, J. A., Bruach, J. M., Palacios, E. and Canals, M. Balance and residence times of ${ }^{210} \mathrm{~Pb}$ and ${ }^{210} \mathrm{Po}$ in surface waters of the northwestern Mediterranean Sea. Cont. Shelf Res. 22(15), 2127-2146 (2002).
27. Broecker, W. S., Goddard, J. and Sarmiento, J. L. The distribution of ${ }^{226} \mathrm{Ra}$ in the Atlantic Ocean. Earth Planet. Sci. Lett. 32, 220-235 (1976).

28. Chung, Y. C. A deep ${ }^{226}$ Ra maximum in the northeast Pacific. Earth Planet. Sci. Lett. 32, 249-257 (1976).

29. Chung, Y. C. ${ }^{226} \mathrm{Ra}$ in the western Indian Ocean. Earth Planet. Sci. Lett. 85, 11-27 (1987).

30. Chung, Y. C. and Craig, H. ${ }^{226}$ Ra in the Pacific Ocean. Earth Planet. Sci. Lett. 49, 267-292 (1980).

31. Ku, T.-L., Huh, C. A. and Chen, P. S. Meridional distribution of ${ }^{226} \mathrm{Ra}$ in the eastern pacific along GEOSECS cruise tracks. Earth Planet. Sci. Lett. 49, 293-308 (1980).

32. Ku, T.-L., Li, Y. H., Mathieu, G. G. and Wong, H. K. Radium in the Indian-Antarctic Ocean South of Australia. J. Geophys. Res. 75(27), 5286-5292 (1970).

33. $\mathrm{Ku}$, T.-L. and Lin, M.-C. ${ }^{226} \mathrm{Ra}$ distribution in the Antarctic Ocean. Earth Planet. Sci. Lett. 32, 236-248 (1976).

34. Ku, T.-L. and Luo, S. New appraisal of Radium-226 as a large-scale oceanic mixing tracer. J. Geophys. Res. 99(C5), 10255-10273 (1994).

35. Charette, M. A., Gonnea, M. E., Morris, P., Statham, P., Fones, G., Planquette, H., Salter, I. and Naveira Garabato, A. Radium as tracers of iron sources fueling a Southern Ocean phytoplankton bloom. Deep-Sea Res. II 54, 1989-1998 (2007).

36. Kaufman, A., Trier, R. M., Broecker, W. S. and Feely, H. W. Distribution of ${ }^{228} \mathrm{Ra}$ in the World Ocean. J. Geophys. Res. 78(36), 8827-8848 (1973).

37. Key, R. M., Stallard, R. F., Moore, W. S. and Sarmiento, J. L. Distribution and flux of ${ }^{226} \mathrm{Ra}$ and ${ }^{228} \mathrm{Ra}$ in the Amazon River estuary. J. Geophys. Res. 90(C4), 6995-7004 (1985).

38. Moore, W. S. Oceanic concentrations of 228 radium. Earth Planet. Sci. Lett. 6, 437-446 (1969).

39. Moore, W. S. Determining coastal mixing rates using radium isotopes. Cont. Shelf Res. 20, 1993-2007 (2000).

40. Moore, W. S. and Santschi, P. H. Ra-228 in the deep Indian Ocean. Deep-Sea Res. 33(1), 107-120 (1986).

41. Moore, W. S., Feely, H. W. and Li, Y. H. Radium isotopes in subarctic waters. Earth Planet. Sci. Lett. 49, 329-340 (1980).

42. Rutgers van der Loeff, M. M., Key, R. M., Scholten, J., Bauch, D. and Michel, A. ${ }^{228}$ Ra as a tracer for shelf water in the Arctic Ocean. Deep-Sea Res. II 42(6), 1533-1553 (1995)

43. Charette, M. A., Buesseler, K. O. and Andrews, J. E. Utility of radium isotopes for evaluating the input and transport of groundwater derived nitrogen to a Cape Cod estuary. Limnol. Oceanogr. 46(2), 465-470 (2001).

44. Krest, J. M., Moore, W. S., Gardner, L. R. and Morris, J. Marsh nutrient export supplied by groundwater discharge: evidence from radium measurements. Glob. Biogeochem. Cycles 14, 167-176 (2000).

45. Moore, W. S. Large groundwater inputs to coastal waters revealed by ${ }^{226} \mathrm{Ra}$ enrichments. Nature 380(6575): 612 (1996).

46. Moore, W. S. Ages of continental shelf waters determined from ${ }^{223} \mathrm{Ra}$ and ${ }^{224} \mathrm{Ra}$. J. Geophys. Res. C Oceans 105(C9): 22117-22122 (2000).

47. Windom, H. L., Moore, W. S., Niencheski, L. F. H. and Jahnke, R. A. Submarine groundwater discharge: a 


\section{N. EVANGELIOU ET AL.}

large, previously unrecognized source of dissolved iron to the South Atlantic Ocean. Mar. Chem. 102(3-4), 252-266 (2006).

48. Tsabaris, C., Patiris, D. L., Karageorgis, A. P., Eleftheriou, G., Papadopoulos, V. P., Georgopoulos, D., Papathanassiou, E. and Povinec, P. P. In-situ radionuclide characterization of a submarine groundwater discharge site at Kalogria Bay, Stoupa, Greece. J. Environ. Radioact. (2011).

49. Povinec, P. P. et al. Characterisation of submarine groundwater discharge offshore south-eastern Sicily. J. Environ. Radioact. 89(1), 81-101 (2006).

50. Evangeliou, N., Florou, H., Bokoros, P. and Scoullos, M. Temporal and spatial distribution of ${ }^{137} \mathrm{Cs}$ in Eastern Mediterranean Sea: horizontal and vertical dispersion in two regions. J. Environ. Radioact. 100, 626-636 (2009).

51. Schmidt, S. Impact of the Mediterranean outflow water on particle dynamics in intermediate waters of the Northeast Atlantic, as revealed by ${ }^{234} \mathrm{Th}$ and ${ }^{228} \mathrm{Th}$. Mar. Chem. 100(3-4), 289-298 (2006).

52. Probonas, M. and Kritidis, P. The exposure of the Greek population to gamma radiation of terrestrial origin. Radiat. Prot. Dosim. 46(2), 123-126 (1993).

53. Trabidou, G. Radiological study in radioactive springs' areas. Ph.D. thesis, University of Athens, Athens, Greece (in Greek) (2004).
54. Hwang, D.-W., Kim, G., Lee, Y.-W. and Yang, H.-S. Estimating submarine inputs of groundwater and nutrients to a coastal bay using radium isotopes. Mar. Chem. 96, 61-71 (2005).

55. Mook, W. G. Introduction of Isotope Hydrology and Radioactive Isotopes of Hydrogen, Oxygen and Carbon. Taylor \& Francis, 226 pp. (2006).

56. Scoullos, M. and Riley, J. P. Water circulation in the Gulf of Elefsis, Greece. Thalassia Jugoslavica 14(3/4), 357-370 (1978).

57. Tsabaris, C. Monitoring natural and artificial radioactivity enhancement in the Aegean Sea using floating measuring systems. Appl. Radiat. Isot. 66, 1599-1603 (2008).

58. Adams, J. A. S. Radioactivity in the lithosphere. In: Nuclear Radiation in Geophysics. Israel, H. and Krebs, A., Eds. Springer-Verlag (1952).

59. Tsabaris, C., Evangeliou, N., Fillis, E., Sotiropoulou, M., Patiris, D. L. and Florou, H. Distribution of natural radioactivity in sediment cores in Amvrakikos Gulf (Western Greece) as a part of IAEA's campaign in Adriatic and Ionian Seas. Radiat. Prot. Dosim. (2011) .

60. Buesseler, K. O. et al. An assessment of particulate organic carbon to thorium-234 ratios in the ocean and their impact on the application of ${ }^{234}$ Th as a POC flux proxy. Mar. Chem. 100, 213-233 (2006). 